\title{
PRIMES IN INTERVALS OF BOUNDED LENGTH
}

\author{
ANDREW GRANVILLE
}

To Yitang Zhang, for showing that one can, no matter what

Abstract. The infamous twin prime conjecture states that there are infinitely many pairs of distinct primes which differ by 2 . Until recently this conjecture had seemed to be far out of reach with current techniques. However, in April 2013, Yitang Zhang proved the existence of a finite bound $B$ such that there are infinitely many pairs of distinct primes which differ by no more than $B$. This is a massive breakthrough, making the twin prime conjecture look highly plausible, and the techniques developed help us to better understand other delicate questions about prime numbers that had previously seemed intractable.

Zhang even showed that one can take $B=70000000$. Moreover, a cooperative team, Polymath8, collaborating only online, had been able to lower the value of $B$ to 4680 . They had not only been more careful in several difficult arguments in Zhang's original paper, they had also developed Zhang's techniques to be both more powerful and to allow a much simpler proof (and this forms the basis for the proof presented herein).

In November 2013, inspired by Zhang's extraordinary breakthrough, James Maynard dramatically slashed this bound to 600, by a substantially easier method. Both Maynard and Terry Tao, who had independently developed the same idea, were able to extend their proofs to show that for any given integer $m \geq 1$ there exists a bound $B_{m}$ such that there are infinitely many intervals of length $B_{m}$ containing at least $m$ distinct primes. We will also prove this much stronger result herein, even showing that one can take $B_{m}=e^{8 m+5}$.

If Zhang's method is combined with the Maynard-Tao setup, then it appears that the bound can be further reduced to 246 . If all of these techniques could be pushed to their limit, then we would obtain $B\left(=B_{2}\right)=12$ (or arguably to 6 ), so new ideas are still needed to have a feasible plan for proving the twin prime conjecture.

The article will be split into two parts. The first half will introduce the work of Zhang, Polymath8, Maynard and Tao, and explain their arguments that allow them to prove their spectacular results. The second half of this article develops a proof of Zhang's main novel contribution, an estimate for primes in relatively short arithmetic progressions.

\section{Part 1. Primes in short intervals}

\section{INTRODUCTION}

1.1. Intriguing questions about primes. Early on in our mathematical education we get used to the two basic rules of arithmetic, addition and multiplication.

Received by the editors September 5, 2014.

2010 Mathematics Subject Classification. Primary 11P32.

This article was shortened for final publication and several important references, namely [7, $10,14,16,37,38,41,43,48,53,59,60,61,65,66,67]$, are no longer directly referred to in the text. Nonetheless we leave these references here for the enthusiastic student. 
Then prime numbers are defined, not in terms of what they are, but rather in terms of what they are not (i.e., that they cannot be factored into two smaller integers). This makes them difficult to find, and to work with.

Prime numbers can be seen to occur rather frequently,

$$
2,3,5,7,11,13,17,19,23,29,31,37,41,43,47,53,59,61, \ldots,
$$

but it took a rather clever construction of the ancient Greeks to establish that there really are infinitely many. Looking closely, patterns begin to emerge; for example, primes often come in pairs:

3 and $5 ; 5$ and $7 ; 11$ and $13 ; 17$ and $19 ; 29$ and $31 ; 41$ and $43 ; 59$ and $61, \ldots$.

One might guess that there are infinitely many such prime pairs. But this is an open, elusive question: the twin prime conjecture. Until recently there was little theoretical evidence for it. A lot of data suggests that these pairs never quit, and the higher view that it fits like the central piece of an enormous jigsaw of conjectures of all sorts of prime patterns. If the twin prime conjecture were false, then one would have to be sceptical of all these conjectures, and our intellectual world would be the poorer for it.

The twin prime conjecture is intriguing to amateur and professional mathematicians alike. It asks for a very delicate additive property of a sequence defined by its multiplicative properties, which some might argue makes it an artificial question. Indeed, number theorists had struggled to identify an approach to this question that captured its essence enough to allow us to make headway. But recently an approach has been found that puts the question firmly within the framework of sieve theory, which has allowed the proof of important steps toward the eventual resolution of the twin prime conjecture (and its generalizations).

In the first few sections we take a leisurely stroll through the historical and mathematical background, so as to give the reader a sense of the great theorems that have been proved recently, from a perspective that will prepare the reader for the details of the proof.

1.2. Other patterns. Staring at the list of primes above, we find four primes which have all the same digits, except the last one:

$11,13,17$, and 19 ; which is repeated with $101,103,107,109$; then $191,193,197,199$;

and one can find many more such examples - are there infinitely many? More simply, how about prime pairs with difference 4 :

3 and $7 ; 7$ and $11 ; 13$ and $17 ; 19$ and $23 ; 37$ and $41 ; 43$ and $47 ; 67$ and $71, \ldots$; or difference 10:

3 and $13 ; 7$ and $17 ; 13$ and $23 ; 19$ and $29 ; 31$ and $41 ; 37$ and $47 ; 43$ and $53, \ldots ?$

Are there infinitely many such pairs? Such questions were probably asked back to antiquity, but the first clear mention of twin primes in the literature appears in a presentation by Alphonse de Polignac, a student at the École Polytechnique in Paris, in 1849. In his honour we now call any integer $h$, for which there are infinitely many prime pairs $p, p+h$, a de Polignac number 1

\footnotetext{
${ }^{1}$ De Polignac also required that $p$ and $p+h$ be consecutive primes, though this requirement is not essential to our discussion here. De Polignac's article [54] is very much that of an amateur
} 
Then there are the Sophie Germain pairs, primes $p$ and $q:=2 p+1$, which prove useful in several simple algebraic constructions:2

2 and $5 ; 3$ and $7 ; 5$ and $11 ; 11$ and $23 ; 23$ and $47 ; 29$ and $59 ; 41$ and $83 ; \ldots$.

Can one predict which prime patterns can occur and which do not? Let's start with differences between primes: One of any two consecutive integers must be even, and so can be prime only if it equals 2 . Hence there is just the one pair, 2 and 3 , of primes with difference 1 . One can make a similar argument for prime pairs with odd difference. Hence if $h$ is an integer for which there are infinitely many prime pairs of the form $p, q=p+h$, then $h$ must be even. We discussed examples for $h=2$, for $h=4$, and for $h=10$ above, and the reader can similarly construct lists of examples for $h=6$ and for $h=8$, and indeed for any other even $h$ that takes her or his fancy. This leads us to bet on the generalized twin prime conjecture, which states that for any even integer $2 k$ there are infinitely many prime pairs $p, q=p+2 k$.

What about prime triples? or quadruples? We saw two examples of prime quadruples of the form $10 n+1,10 n+3,10 n+7,10 n+9$, and we believe that there are infinitely many. What about other patterns? Evidently any pattern that includes an odd difference cannot succeed. Are there any other obstructions? The simplest pattern that avoids an odd difference is $n, n+2, n+4$. One finds the one example 3, 5, 7 of such a prime triple, but no others. Further examination makes it clear why not: One of the three numbers is always divisible by 3 . This is analogous to one of $n, n+1$ being divisible by 2 ; and, similarly, one of $n, n+6, n+12, n+$ $18, n+24$ is always divisible by 5 . The general obstruction can be described as follows:

For a given set of distinct integers $a_{1}<a_{2}<\cdots<a_{k}$, we say that prime $p$ is an obstruction if $p$ divides at least one of $n+a_{1}, \ldots, n+a_{k}$, for every integer $n$. In other words, $p$ divides

$$
\mathcal{P}(n)=\left(n+a_{1}\right)\left(n+a_{2}\right) \cdots\left(n+a_{k}\right)
$$

for every integer $n$, which can be classified by the condition that the set $a_{1}, a_{2}, \ldots, a_{k}$ $(\bmod p)$ includes all of the residue classes $\bmod p$. If no prime is an obstruction, then we say that $x+a_{1}, \ldots, x+a_{k}$ is an admissible set of forms 3

In 1904 Dickson made the optimistic conjecture that if there is no such obstruction to a set of linear forms being infinitely often prime, then they are infinitely often simultaneously prime. That is:

Conjecture. If $x+a_{1}, \ldots, x+a_{k}$ is an admissible set of forms, then there are infinitely many integers $n$ such that $n+a_{1}, \ldots, n+a_{k}$ are all prime numbers.

mathematician, developing a first understanding of the sieve of Eratosthenes. His other "conjecture" in the paper, asking whether every odd number is the sum of a prime and power of two, is false for as small an example as 127 .

${ }^{2}$ The group of reduced residues $\bmod q$ is a cyclic group of order $q-1=2 p$, and therefore isomorphic to $C_{2} \times C_{p}$ if $p>2$. Hence, the order of each element in the group is either 1 (that is, $1(\bmod q)), 2($ that is, $-1(\bmod q)), p($ the squares $\bmod q)$, or $2 p=q-1$. Hence, $g(\bmod q)$ generates the group of reduced residues if and only if $g$ is not a square $\bmod q$ and $g \not \equiv-1(\bmod q)$.

${ }^{3}$ Notice that $a_{1}, a_{2}, \ldots, a_{k}(\bmod p)$ can occupy no more than $k$ residue classes $\bmod p$, and so if $p>k$, then $p$ cannot be an obstruction. Hence, to check whether a given set $A$ of $k$ integers is admissible, one needs only find one residue class $b_{p}(\bmod p)$, for each prime $p \leq k$, which does not contain any element of $A$. 
In this case, we call $n+a_{1}, \ldots, n+a_{k}$ a $k$-tuple of prime numbers. Dickson's prime $k$-tuple conjecture states that if a set $b_{1} x+a_{1}, \ldots, b_{k} x+a_{k}$ of linear forms is admissible (that is, if the forms are all positive at infinitely many integers $x$ and for each prime $p$ there exists an integer $n$ such that $\left.p \nmid \mathcal{P}(n):=\prod_{j}\left(b_{j} n+a_{j}\right)\right)$, then there are infinitely many integers $n$ for which $b_{1} n+a_{1}, \ldots, b_{k} n+a_{k}$ are all primes.

To date, this has not been proven for any $k>1$; however, following Zhang's work, we begin to get close for $k=2$. Indeed, Zhang has proved a weak variant of this conjecture for $k=2$, as we shall see. Moreover, Maynard [45] and Tao 63] have gone on to prove a weak variant for any $k \geq 2$.

The above conjecture can be extended to linear forms in more than one variable (for example the set of forms $m, m+n, m+4 n$ ):

The prime $k$-tuplets conjecture: If a set of $k$ linear forms in $n$ variables is admissible, then there are infinitely many sets of $n$ integers such that when we substitute these integers into the forms, we get a $k$-tuple of prime numbers.

There has been substantial recent progress on this conjecture. The famous breakthrough was Green and Tao's theorem [29] for the $k$-tuple of linear forms in the two variables $a$ and $d$ :

$$
a, a+d, a+2 d, \ldots, a+(k-1) d
$$

(in other words, there are infinitely many $k$-term arithmetic progressions of primes). Along with Ziegler in [30, they went on to prove the prime $k$-tuplets conjecture for any admissible set of linear forms, provided that no two satisfy a linear equation over the integers. What a remarkable theorem! Unfortunately, these exceptions include many of the questions we are most interested in: for example, $p, q=p+2$ satisfy the linear equation $q-p=2$; and $p, q=2 p+1$ satisfy the linear equation $q-2 p=1$.

Finally, we also believe that the conjecture holds if we consider any admissible set of $k$ irreducible polynomials with integer coefficients, with any number of variables. For example we believe that $n^{2}+1$ is infinitely often prime and that there are infinitely many prime triples $m, n, m^{2}-2 n^{2}$.

1.3. The new results; primes in bounded intervals. In this section we state Zhang's main theorem, as well as the improvement of Maynard and Tao, and we discuss a few of the more beguiling consequences.

Zhang's main theorem. There exists an integer $k$ such that if $x+a_{1}, \ldots, x+a_{k}$ is an admissible set of forms, then there are infinitely many integers $n$ for which at least two of $n+a_{1}, \ldots, n+a_{k}$ are prime numbers.

Note that the result states that only two of the $n+a_{i}$ are prime, not all (as would be required in the prime $k$-tuplets conjecture). Zhang proved this result for a fairly large value of $k$, that is $k=3500000$, which has been reduced to $k=105$ by Maynard, and now to $k=50$ in [58. Of course if one could take $k=2$, then we would have the twin prime conjecture 4 but the most optimistic plan at the moment, along the lines of Zhang's proof, would yield $k=3$ (see Section 8.1).

\footnotetext{
${ }^{4}$ And the generalized twin prime conjecture, and that there are infinitely many Sophie Germain pairs (if one could use nonmonic polynomials), and ....
} 
To deduce that there are bounded gaps between primes from Zhang's theorem we need only show the existence of an admissible set with $k$ elements. This is not difficult - simply let the $a_{i}$ be the first $k$ primes $>k 5$ Hence we have proved:

Corollary 1.1 (Bounded gaps between primes). There exists a bound B such that there are infinitely many integers pairs of prime numbers $p<q<p+B$.

Finding the narrowest admissible $k$-tuples is a challenging question. The prime number theorem together with our construction above implies that $B \leq k(\log k+C)$ for some constant $C$, but it is interesting to get better bounds. For Maynard's $k=105$, Engelsma exhibited an admissible 105-tuple of width 600 and proved that there are no narrower ones. The narrowest 50-tuple has width 246 , and one such tuple is:

$0,4,6,16,30,34,36,46,48,58,60,64,70,78,84,88,90,94,100,106$, $108,114,118,126,130,136,144,148,150,156,160,168,174,178,184$, $190,196,198,204,210,214,216,220,226,228,234,238,240,244,2466$

Corollary 1.1 further implies (for $B=246$ )

Corollary 1.2. There is an integer $h, 0<h \leq B$, such that there are infinitely many pairs of primes $p, p+h$.

That is, some positive integer $\leq B$ is a de Polignac number. In fact one can go a little further using Zhang's main theorem and deduce that if $A$ is any admissible set of $k$ integers, then there is an integer $h \in(A-A)^{+}:=\{a-b: a>b \in A\}$ such that there are infinitely many pairs of primes $p, p+h$. One can find many beautiful consequences of this: for example, a positive proportion of even integers are de Polignac numbers.

Zhang's theorem can be proved for $k$-tuplets $b_{1} x+a_{1}, \ldots, b_{k} x+a_{k}$ with minor (and obvious) modifications to the proof given herein.

Next we state the theorem of Maynard and of Tao:

The Maynard-Tao theorem. For any given integer $m \geq 2$, there exists an integer $k$ such that if $x+a_{1}, \ldots, x+a_{k}$ is an admissible set of forms, then there are infinitely many integers $n$ for which at least $m$ of $n+a_{1}, \ldots, n+a_{k}$ are prime numbers.

This includes and extends Zhang's theorem (which is the case $k=2$ ). The proof even allows one to make this explicit (we will obtain $k \leq e^{8 m+4}$, and Maynard improves this to $k \leq \mathrm{cm}^{2} e^{4 m}$ for some constant $c>0$ ).

Corollary 1.3 (Bounded intervals with $m$ primes). For any given integer $m \geq 2$, there exists a bound $B_{m}$ such that there are infinitely many intervals $\left[x, x+B_{m}\right]$ (with $x \in \mathbb{Z}$ ) which contain $m$ prime numbers.

We will prove that one can take $B_{m}=e^{8 m+5}$ (which Maynard improves to $B_{m}=c m^{3} e^{4 m}$, and the Polymath team [58] to $B_{m}=c m e^{\left(4-\frac{28}{157}\right) m}$, for some constant $c>0$ ).

A Dickson $k$-tuple is a set of integers $a_{1}<\cdots<a_{k}$ such that there are infinitely many integers for which $n+a_{1}, n+a_{2}, \ldots, n+a_{k}$ are each prime.

\footnotetext{
${ }^{5}$ This is admissible since none of the $a_{i}$ is $0(\bmod p)$ for any $p \leq k$, and the $p>k$ were handled in footnote 3 .

${ }^{6}$ Sutherland's website http://math.mit.edu/ primegaps/ lists narrowest $k$-tuples for all small $k$.
} 
Corollary 1.4. A positive proportion of $m$-tuples of integers are Dickson $m$-tuples.

Proof. With the notation as in the Maynard-Tao theorem, let $R=\prod_{p \leq k} p$, select $x$ to be a large integer multiple of $R$, and let $\mathcal{N}:=\{n \leq x:(n, R)=1\}$ so that $|\mathcal{N}|=\frac{\phi(R)}{R} x$. Any subset of $k$ elements of $\mathcal{N}$ is admissible, since it does not contain any integer $\equiv 0(\bmod p)$ for each prime $p \leq k$. There are $\left(\begin{array}{c}|\mathcal{N}| \\ k\end{array}\right)$ such $k$-tuples. Each contains a Dickson $m$-tuple by the Maynard-Tao theorem.

Now suppose that there are $T(x)$ Dickson $m$-tuples that are subsets of $\mathcal{N}$. Any such $m$-tuple is a subset of exactly $\left(\begin{array}{c}|\mathcal{N}|-m \\ k-m\end{array}\right)$ of the $k$-subsets of $\mathcal{N}$, and hence

$$
T(x) \cdot\left(\begin{array}{c}
|\mathcal{N}|-m \\
k-m
\end{array}\right) \geq\left(\begin{array}{c}
|\mathcal{N}| \\
k
\end{array}\right)
$$

and therefore $T(x) \geq(|\mathcal{N}| / k)^{m}=\left(\frac{\phi(R)}{R} / k\right)^{m} \cdot x^{m}$, as desired.

This proof yields that, as a proportion of the $m$-tuples in $\mathcal{N}$,

$$
T(x) /\left(\begin{array}{c}
|\mathcal{N}| \\
m
\end{array}\right) \geq 1 /\left(\begin{array}{c}
k \\
m
\end{array}\right) .
$$

The $m=2$ case implies that at least $\frac{1}{5460}$ th of the even integers are de Polignac numbers. (This is improved to at least $\frac{1}{177}$ in [27], which also discusses limitations on what can be deduced from a result like Zhang's theorem.)

Zhang's theorem and the Maynard-Tao theorem each hold for any admissible $k$-tuple of linear forms (not just those of the form $x+a$ ). With this we can prove several other amusing consequences:

- Corollary 1.2 holds if we insist that the primes in the Dickson $k$-tuples are consecutive primes.

- There exists a constant $H$ such that every interval $[x, x+H]$ contains a de Polignac number (see [51]).

- There are infinitely many $m$-tuples of consecutive primes such that each pair in the $m$-tuple differ from one another by just two digits when written in base 10 .

- For any $m \geq 2$ and coprime integers $a$ and $q$, there are infinitely many intervals $\left[x, x+q B_{m}\right]$ (with $x \in \mathbb{Z}$ ) which contain exactly $m$ prime numbers, each $\equiv a$ $(\bmod q) .7$

- For any integer $r \geq 2$, there are infinitely many $m$-tuples of distinct primes $q_{1}, \ldots, q_{m}$, such that the ratios $\left(q_{i}-1\right) /\left(q_{j}-1\right)$ are all (bounded) powers of $r$.

- Let $d_{n}=p_{n+1}-p_{n}$ where $p_{n}$ is the $n$th smallest prime. Fix $m \geq 1$. There are infinitely many $n$ for which $d_{n}<d_{n+1}<\cdots<d_{n+m}$. There are also infinitely many $n$ for which $d_{n}>d_{n+1}>\cdots>d_{n+m}$ (see [2]). This was a favorite problem of Paul Erdős, though we do not see how to deduce such a result for other orderings of the $d_{n} \frac{8}{8}$

- One can also deduce [56] that there are infinitely many $n$ such that $s_{r}\left(p_{n}\right)<$ $s_{r}\left(p_{n+1}\right)<\cdots<s_{r}\left(p_{n+m}\right)$, where $s_{r}(N)$ denotes the sum of the digits of $N$ when written in base $r$ (as well as $\left.s_{r}\left(p_{n}\right)>\cdots>s_{r}\left(p_{n+m}\right)\right)$.

\footnotetext{
${ }^{7}$ Thanks to Tristan Freiberg for pointing this out to me (see also [18]). However, I do not see how to modify the proof to show, given $r_{1}, \ldots, r_{m}$ coprime to $q$, that one has primes $p_{n+1}, \ldots, p_{n+m}$ with $p_{n+j} \equiv r_{j}(\bmod q)$ for $j=1, \ldots, m$.

${ }^{8}$ It was also shown in 2] that the $d_{n+j}$ can grow as fast as one likes. Moreover, one can insist that $d_{n}\left|d_{n+1}\right| \cdots \mid d_{n+m}$.
} 
- Moreover [56], there are infinitely many $n$ such that $\phi\left(p_{n}-1\right)<\phi\left(p_{n+1}-1\right)<$ $\cdots<\phi\left(p_{n+m}-1\right)$ (as well as $\left.\phi\left(p_{n}-1\right)>\cdots>\phi\left(p_{n+m}-1\right)\right)$. An analogous result holds with $\phi$ replaced by $\sigma, \tau, \nu$ and many other arithmetic functions.

- If $\alpha$ is an algebraic, irrational number, then by [9] there are infinitely many $n$ such that at least $m$ of $[\alpha n],[\alpha(n+1)], \ldots,[\alpha(n+k)]$ are prime (where $[t]$ denotes the integer part of $t$ ). This result can be extended to any irrational number $\alpha$ for which there exists $r$ such that $|p \alpha-q| \geq 1 / p^{r}$ for all integers $p, q>0$.

In the eight months since Maynard's preprint, many further interesting applications of his technique have appeared, some of which we discuss in Section 12 .

1.4. Bounding the gaps between primes: a brief history. The young Gauss, examining Chernac's table of primes up to one million, guessed that "the density of primes at around $x$ is roughly $1 / \log x "$. This was subsequently shown to be true, as a consequence of the prime number theorem. Therefore, we are guaranteed that there are infinitely many pairs of primes $p<q$ for which $q-p \leq(1+\epsilon) \log p$ for any fixed $\epsilon>0$, which is not quite as small a gap as we are hoping for! Nonetheless, this raises the question: Fix $c>0$. Can we even prove:

There are infinitely many pairs of primes $p<q$ with $q<p+c \log p$ ?

This follows for all $c>1$ by the prime number theorem, but it is not easy to prove such a result for any particular value of $c \leq 1$. The first unconditional result bounding gaps between primes for some $c<1$, was proved by Erdős in 1940 using the small sieve. In 1966, Bombieri and Davenport 4 used the BombieriVinogradov theorem to prove this for any $c \geq \frac{1}{2}$. In 1988 Maier 44] observed that one can easily modify this to obtain any $c \geq \frac{1}{2} e^{-\gamma}$. He further improved this by combining the approaches of Erdös and of Bombieri and Davenport to obtain some bound a little smaller than $\frac{1}{4}$, in a technical tour de force.

The first big breakthrough occurred in 2005 when Goldston, Pintz, and Ylldirım [23] were able to show that there are infinitely many pairs of primes $p<q$ with $q<p+c \log p$, for any given $c>0$. Indeed, they extended their methods to show that, for any $\epsilon>0$, there are infinitely many pairs of primes $p<q$ for which

$$
q-p<(\log p)^{1 / 2+\epsilon}
$$

It is their method that forms the basis of the discussion in this paper.

The earliest results on short gaps between primes were proved assuming the Generalized Riemann Hypothesis. Later unconditional results, starting with Bombieri and Davenport, used the Bombieri-Vinogradov theorem in place of the Generalized Riemann Hypothesis. It is surprising that these tools appear in arguments about gaps between primes, since they are formulated to better understand the distribution of primes in arithmetic progressions.

Like Bombieri and Davenport, Goldston, Pintz, and Yıldırım showed that one can better understand small gaps between primes by obtaining strong estimates on primes in arithmetic progressions, as in the Bombieri-Vinogradov theorem. Even more, by assuming a strong, but widely believed, conjecture about the equidistribution of primes in arithmetic progressions, which extends the BombieriVinogradov theorem, one can prove Zhang's theorem with $k=5$. Applying this result to the admissible 5-tuple, $\{0,2,6,8,12\}$ implies that there are infinitely many pairs of primes $p<q$ which differ by no more than 12 ; that is, there exists a positive, even integer $2 k \leq 12$ such that there are infinitely pairs of primes $p, p+2 k$. 
After Goldston, Pintz, and Yıldırım, most of the experts tried and failed to obtain enough of an improvement of the Bombieri-Vinogradov theorem to deduce the existence of some finite bound $B$ such that there are infinitely many pairs of primes that differ by no more than $B$. To improve the Bombieri-Vinogradov theorem is no mean feat, and people have longed discussed "barriers" to obtaining such improvements. In fact a technique to improve the Bombieri-Vinogradov theorem had been developed by Fouvry [15, and by Bombieri, Friedlander, and Iwaniec [5], but this was neither powerful enough nor general enough to work in this circumstance.

Enter Yitang Zhang, an unlikely figure to go so much further than the experts and to find exactly the right improvement and refinement of the Bombieri-Vinogradov theorem to establish the existence of the elusive bound $B$ such that there are infinitely many pairs of primes that differ by no more than $B$. By all accounts, Zhang was a brilliant student in Beijing from 1978 to the mid-1980s, finishing with a master's degree, and then working on the Jacobian conjecture for his Ph.D. at Purdue, graduating in 1992. He did not proceed to a job in academia, working in odd jobs, such as in a sandwich shop, at a motel, and as a delivery worker. Finally in 1999 he got a job at the University of New Hampshire as a lecturer. From time to time a lecturer devotes their energy to working on proving great results, but few have done so with such aplomb as Zhang. Not only did he prove a great result, but he did so by improving technically on the experts, having important key ideas that they missed and by developing a highly ingenious and elegant construction concerning exponential sums. Then, so as not to be rejected out of hand, he wrote his difficult paper up in such a clear manner that it could not be denied. Albert Einstein worked in a patent office, Yitang Zhang in a Subway sandwich shop; both found time, despite the unrelated calls on their time and energy, to think the deepest thoughts in science. Moreover, Zhang's breakthrough came at the relatively advanced age of 57 . Truly extraordinary.

After Zhang, a group of researchers decided to team up online to push those techniques created by Zhang to their limit. This was the eighth incarnation of the Polymath project, which is an experiment to see whether this sort of collaboration can help research develop beyond the traditional boundaries set by our academic culture. The original bound of 70,000,000 was quickly reduced and, seemingly every few weeks, different parts of Zhang's argument could be improved, so that the bound came down to the thousands. Moreover, the Polymath 8 researchers found variants on Zhang's argument about the distribution of primes in arithmetic progressions that allow one to avoid some of the deeper ideas that Zhang used. These modifications enabled your author to give an accessible complete proof in this article.

After these clarifications of Zhang's work, two researchers asked themselves whether the original "setup" of Goldston, Pintz, and Yıldırım could be modified to get better results. James Maynard obtained his Ph.D. this summer at Oxford, writing one of the finest theses in sieve theory of recent years. His thesis work equipped him perfectly to question whether the basic structure of the proof could be improved. Unbeknownst to Maynard, at much the same time (late October), one of the world's greatest living mathematicians, Terry Tao, asked himself the same question. Both found, to their surprise, that a relatively minor variant made an enormous difference, and that it was suddenly much easier to prove Zhang's Main Theorem and to go far beyond, because one can avoid having to prove any 
difficult new results about primes in arithmetic progressions. Moreover, it is now not difficult to prove results about $m$ primes in a bounded interval, rather than just two.

\section{The Distribution of PRIMES, DIVISORS, AND PRIME $k$-TUPLETS}

2.1. The prime number theorem. As we mentioned in the previous section, Gauss observed, at the age of 16 , that "the density of primes at around $x$ is roughly $1 / \log x "$, which leads quite naturally to the conjecture that

$$
\#\{\text { primes } p \leq x\} \approx \int_{2}^{x} \frac{d t}{\log t} \sim \frac{x}{\log x} \quad \text { as } x \rightarrow \infty .
$$

(We use the symbol $A(x) \sim B(x)$ for two functions $A$ and $B$ of $x$, to mean that $A(x) / B(x) \rightarrow 1$ as $x \rightarrow \infty$.) This, the prime number theorem, was proved in 1896, and the integral provides a considerably more precise approximation to the number of primes $\leq x$ than $x / \log x$. However, this integral is rather cumbersome to work with, and so it is natural to instead weight each prime with $\log p$; that is, we work with

$$
\Theta(x):=\sum_{p \text { prime }} \log p,
$$

and the prime number theorem is equivalent to

$$
\Theta(x) \sim x \quad \text { as } x \rightarrow \infty .
$$

2.2. The prime number theorem for arithmetic progressions, I. Any prime divisor of $(a, q)$ is an obstruction to the primality of values of the polynomial $q x+a$, and these are the only such obstructions. The prime $k$-tuplets conjecture therefore implies that if $(a, q)=1$, then there are infinitely many primes of the form $q n+a$. This was first proved by Dirichlet in 1837. Once proved, one might ask for a more quantitative result. If we look at the primes in the arithmetic progressions $(\bmod 10)$ :

$11,31,41,61,71,101,131,151,181,191,211,241, \ldots$,

$3,13,23,43,53,73,83,103,113,163,173,193,223,233, \ldots$,

$7,17,37,47,67,97,107,127,137,157,167,197,227, \ldots$,

$19,29,59,79,89,109,139,149,179,199,229,239, \ldots$,

then there seem to be roughly equal numbers in each, and this pattern persists as we look further out. Let $\phi(q)$ denote the number of $a(\bmod q)$ for which $(a, q)=1$ (which are the only arithmetic progressions in which there can be more than one prime), and so we expect that

$$
\Theta(x ; q, a):=\sum_{\substack{p \operatorname{prime} \\ p \leq x \\ p \equiv a}} \log p \sim \frac{x}{\phi(q)} \text { as } x \rightarrow \infty .
$$

This is the prime number theorem for arithmetic progressions and was first proved by suitably modifying the proof of the prime number theorem.

The function $\phi(q)$ was studied by Euler, who showed that it is multiplicative, that is

$$
\phi(q)=\prod_{p^{e} \| q} \phi\left(p^{e}\right)
$$


(where $p^{e} \| q$ means that $p^{e}$ is the highest power of prime $p$ dividing $q$ ), and from this formula $\phi(q)$ can easily be determined for all $q$ since $\phi\left(p^{e}\right)=p^{e}-p^{e-1}$ for all $e \geq 1$.

2.3. The prime number theorem and the Möbius function. Multiplicative functions lie at the heart of much of the theory of the distribution of prime numbers. One in particular, the Möbius function, $\mu(n)$, plays a prominent role. It is defined as $\mu(p)=-1$ for every prime $p$, and $\mu\left(p^{m}\right)=0$ for every prime $p$ and exponent $m \geq 2$; the value at any given integer $n$ is then deduced from the values at the prime powers, by multiplicativity: If $n$ is square-free, then $\mu(n)$ equals 1 or -1 according to whether $n$ has an even or odd number of prime factors, respectively. One might guess that there are roughly equal numbers of each, which one can phrase as the conjecture that

$$
\frac{1}{x} \sum_{n \leq x} \mu(n) \rightarrow 0 \text { as } n \rightarrow \infty .
$$

This is a little more difficult to prove than it looks; indeed it is also equivalent to (2.1), the prime number theorem. That equivalence is proved using the remarkable identity

$$
\sum_{a b=n} \mu(a) \log b= \begin{cases}\log p & \text { if } n=p^{m}, \text { where } p \text { is prime, } m \geq 1 \\ 0 & \text { otherwise. }\end{cases}
$$

For more on this connection see the forthcoming book [28].

2.4. Recognizing prime powers and prime $k$-tuplets. It is convenient to denote the right-hand side of (2.2) by $\Lambda(n)$ so that

$$
\Lambda(n)=\sum_{d \mid n} \mu(d) \log n / d .
$$

In (2.2) we saw that $\Lambda(n)$ is supported (only) on integers $n$ that are prime powers 9 so this identity allows us to distinguish between composites and prime powers. This is useful because the functions in the summands are arithmetic functions that can be studied directly. Such identities can be used to identify integers with no more than $k$ prime factors. For example

$$
\Lambda_{2}(n):=\sum_{d \mid n} \mu(d)(\log n / d)^{2}= \begin{cases}(2 m-1)(\log p)^{2} & \text { if } n=p^{m} \\ 2 \log p \log q & \text { if } n=p^{a} q^{b}, p \neq q \\ 0 & \text { otherwise }\end{cases}
$$

that is, $\Lambda_{2}(n)$ is supported (only) on integers $n$ that have no more than two distinct prime factors. In general (as seems to have first been discovered by Golomb [25]),

$$
\Lambda_{k}(n):=\sum_{d \mid n} \mu(d)(\log n / d)^{k}
$$

is supported only when $n$ has no more than $k$ distinct prime factors (that is, $\Lambda_{k}(n)=$ 0 if $\nu(n)>k$, where $\nu(m)$ denotes the number of distinct prime factors of $m)$. One

\footnotetext{
${ }^{9}$ By "supported on" we mean "can be nonzero only on".
} 
can deduce (what at first sight seems to be a generalization) that, for any integer $R$,

$$
\sum_{d \mid n} \mu(d)(\log R / d)^{k}
$$

is supported only when $n$ has no more than $k$ distinct prime factors.

Suppose that $0<a_{1}<\cdots<a_{k}$. We now show that if $n \geq a_{k}^{k-1}$ and $\Lambda_{k}(\mathcal{P}(n)) \neq$ 0 , then $\mathcal{P}(n)$ must have exactly $k$ distinct prime factors; moreover, if the $k$ prime factors of $\mathcal{P}(n)$ are $p_{1}, \ldots, p_{k}$, then

$$
\Lambda_{k}(\mathcal{P}(n))=k !\left(\log p_{1}\right) \cdots\left(\log p_{k}\right) .
$$

Proof. If $\mathcal{P}(n)$ has $r \leq k-1$ distinct prime factors, call them $p_{1}, \ldots, p_{r}$. For each $p_{i}$ select some index $j=j(i)$ for which the power of $p_{i}$ dividing $n+a_{j}$ is maximized. Evidently there exists some $J, 1 \leq J \leq k$, which is not a $j(i)$ for any $i$. Therefore, if $p_{i}^{e_{i}} \| n+a_{J}$ for each $i$, then

$$
p_{i}^{e_{i}} \mid\left(n+a_{J}\right)-\left(n+a_{j(i)}\right)=\left(a_{J}-a_{j(i)}\right), \text { which divides } \prod_{\substack{1 \leq j \leq k \\ j \neq j}}\left(a_{J}-a_{j}\right) .
$$

Hence

$$
n+a_{J}=\prod_{i=1}^{r} p_{i}^{e_{i}} \text { divides } \prod_{\substack{1 \leq j \leq k \\ j \neq J}}\left(a_{J}-a_{j}\right),
$$

and so $n<n+a_{J} \leq a_{k}^{k-1} \leq n$, by hypothesis, which is impossible.

Selberg championed a surprising, yet key, notion of sieve theory: the truncation

$$
\sum_{\substack{d \mid n \\ d \leq R}} \mu(d) \log R / d
$$

is "sensitive to primes" (though not necessarily only supported on integers with few prime factors), and it is considerably easier to work with in various analytic arguments. In our case, we will work with the function

$$
\sum_{\substack{d \mid \mathcal{P}(n) \\ d \leq R}} \mu(d)(\log R / d)^{k}
$$

which is analogously "sensitive" to prime $k$-tuplets and easier to work with than the full sum for $\Lambda_{k}(\mathcal{P}(n))$.

2.5. A quantitative prime $k$-tuplets conjecture. We are going to develop a heuristic to "guesstimate" the number of pairs of twin primes $p, p+2$ up to $x$. We start with Gauss's statement that "the density of primes at around $x$ is roughly $1 / \log x "$. Hence, the probability that $p$ is prime is $1 / \log x$, and the probability that $p+2$ is prime is $1 / \log x$, so, assuming that these events are independent, the probability that $p$ and $p+2$ are simultaneously prime is

$$
\frac{1}{\log x} \cdot \frac{1}{\log x}=\frac{1}{(\log x)^{2}},
$$

and so we might expect about $x /(\log x)^{2}$ pairs of twin primes $p, p+2 \leq x$. However, there is a problem with this reasoning, since we are implicitly assuming that the events " $p$ is prime for an arbitrary integer $p \leq x$ " and " $p+2$ is prime for an 
arbitrary integer $p \leq x$ " can be considered to be independent. This is obviously false since, for example, if $p$ is even, then $p+2$ must also be 10 So we correct for the non-independence modulo small primes $q$, by the ratio of the probability that both $p$ and $p+2$ are not divisible by $q$, to the probability that $p$ and $p^{\prime}$ are not divisible by $q$.

Now the probability that $q$ divides an arbitrary integer $p$ is $1 / q$; hence, the probability that $p$ is not divisible by $q$ is $1-1 / q$. Therefore, the probability that both of two independently chosen integers are not divisible by $q$ is $(1-1 / q)^{2}$.

The probability that $q$ does not divide either $p$ or $p+2$, equals the probability that $p \not \equiv 0$ or $-2(\bmod q)$. If $q>2$, then $p$ can be in any one of $q-2$ residue classes $\bmod q$, which occurs, for a randomly chosen $p(\bmod q)$, with probability $1-2 / q$. If $q=2$, then $p$ can be in only one residue class $\bmod 2$, which occurs with probability $1 / 2$. Hence, the "correction factor" for divisibility by 2 is

$$
\frac{\left(1-\frac{1}{2}\right)}{\left(1-\frac{1}{2}\right)^{2}}=2,
$$

and the "correction factor" for divisibility by any prime $q>2$ is

$$
\frac{\left(1-\frac{2}{q}\right)}{\left(1-\frac{1}{q}\right)^{2}}
$$

Divisibility by different small primes is independent, as we vary over values of $n$, by the Chinese Remainder Theorem, and so we might expect to multiply together all of these correction factors, corresponding to each "small" prime $q$. The question then becomes, what does "small" mean? In fact, it does not matter much because the product of the correction factors over larger primes is very close to 1 , and hence we can simply extend the correction to be a product over all primes $q$. (More precisely, the infinite product over all $q$, converges.) Hence, we define the twin prime constant to be

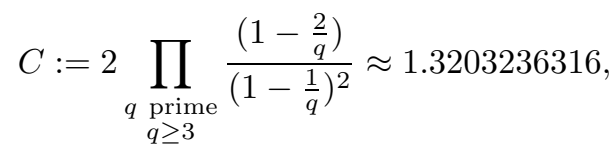

the total correction factor over all primes $q$. We then conjecture that the number of prime pairs $p, p+2 \leq x$ is

$$
\sim C \frac{x}{(\log x)^{2}} .
$$

Computational evidence suggests that this is a pretty good guess. An analogous argument implies the conjecture that the number of prime pairs $p, p+2 k \leq x$ is

$$
\sim C \prod_{\substack{p \mid k \\ p \geq 3}}\left(\frac{p-1}{p-2}\right) \frac{x}{(\log x)^{2}} .
$$

This argument is easily modified to make an analogous prediction for any $k$ tuple: Given $a_{1}, \ldots, a_{k}$, let $\Omega(p)$ be the set of distinct residues given by $a_{1}, \ldots, a_{k}$ $(\bmod p)$, and then let $\omega(p)=|\Omega(p)|$. None of the $n+a_{i}$ is divisible by $p$ if and only

\footnotetext{
${ }^{10}$ This reasoning can be seen to be false for a more dramatic reason: the analogous argument implies that there are $\sim x /(\log x)^{2}$ prime pairs $p, p+1 \leq x$.
} 
if $n$ is in any one of $p-\omega(p)$ residue classes $\bmod p$, and therefore the correction factor for prime $p$ is

$$
\frac{\left(1-\frac{\omega(p)}{p}\right)}{\left(1-\frac{1}{p}\right)^{k}}
$$

Hence, we predict that the number of prime $k$-tuplets $n+a_{1}, \ldots, n+a_{k} \leq x$ is

$$
\sim C(a) \frac{x}{(\log x)^{k}}, \text { where } \quad C(a):=\prod_{p} \frac{\left(1-\frac{\omega(p)}{p}\right)}{\left(1-\frac{1}{p}\right)^{k}} .
$$

An analogous conjecture, via similar reasoning, can be made for the frequency of prime $k$-tuplets of polynomial values in several variables. What is remarkable is that computational evidence suggests that these conjectures do approach the truth, though this rests on the rather shaky theoretical framework given here. A more convincing theoretical framework based on the circle method (so rather more difficult) was given by Hardy and Littlewood [32, which we will discuss in Appendix A.

\section{UNIFORMITY IN ARITHMETIC PROGRESSIONS}

3.1. When primes are first equidistributed in arithmetic progressions. By when are we guaranteed that the primes are more or less equidistributed amongst the arithmetic progressions $a(\bmod q)$ with $(a, q)=1$ ? That is, for what $x$ do we have

$$
\Theta(x ; q, a) \sim \frac{x}{\phi(q)} \text { for all }(a, q)=1 ?
$$

Here $x$ should be a function of $q$, and the asymptotic should hold as $q \rightarrow \infty$.

Calculations suggest that, for any $\epsilon>0$, if $q$ is sufficiently large and $x \geq q^{1+\epsilon}$, then the primes up to $x$ are equidistributed amongst the arithmetic progressions $a$ $(\bmod q)$ with $(a, q)=1$, that is (3.1) holds. However, no one has a plausible plan of how to prove such a result at the moment. The slightly weaker statement that (3.1) holds for any $x \geq q^{2+\epsilon}$ can be shown to be true, assuming the Generalized Riemann Hypothesis. This gives us a clear plan for proving such a result, but one which has seen little progress in the last century!

The best unconditional results known involve much larger values of $x$, equidistribution only being proved once $x \geq e^{q^{\epsilon}}$. This is the Siegel-Walfisz theorem, and it can be stated in several (equivalent) ways with an error term: For any $B>0$, we have

$$
\Theta(x ; q, a)=\frac{x}{\phi(q)}+O\left(\frac{x}{(\log x)^{B}}\right) \text { for all }(a, q)=1 .
$$

Alternatively, we have the statement: For any $A>0$ there exists $B>0$ such that if $q<(\log x)^{A}$, then

$$
\Theta(x ; q, a)=\frac{x}{\phi(q)}\left\{1+O\left(\frac{1}{(\log x)^{B}}\right)\right\} \text { for all }(a, q)=1 .
$$

That $x$ needs to be so large compared to $q$ limits the applicability of this result.

The great breakthough of the second half of the twentieth century came in appreciating that for many applications, it is not so important that we know that equidistribution holds for every $a$ with $(a, q)=1$ and every $q$ up to some $Q$, but rather that it holds for most such $q$ (with $Q=x^{1 / 2-\epsilon}$ ). It takes some juggling of 
variables to state the Bombieri-Vinogradov theorem: We are interested, for each modulus $q$, in the size of the largest error term

$$
\max _{\substack{a \bmod q \\(a, q)=1}}\left|\Theta(x ; q, a)-\frac{x}{\phi(q)}\right|
$$

or even

$$
\max _{y \leq x} \max _{\substack{a \bmod q \\(a, q)=1}}\left|\Theta(y ; q, a)-\frac{y}{\phi(q)}\right| .
$$

The bounds $-\frac{x}{\phi(q)} \leq \Theta(x ; q, a)-\frac{x}{\phi(q)} \leq\left(\frac{x}{q}+1\right) \log x$ are trivial, the upper bound obtained by bounding the possible contribution from each term of the arithmetic progression. We would like to improve on these bounds, perhaps by a power of $\log x$ (as in (3.2), but we are unable to do so for all $q$. However, what we can prove is that exceptional $q$ are few and far between 11 and the Bombieri-Vinogradov theorem expresses this in a useful form. The "trivial" upper bound, obtained by adding up the above quantities over all $q \leq Q<x$, is

$$
\sum_{q \leq Q} \max _{a}\left|\Theta(x ; q, a)-\frac{x}{\phi(q)}\right| \leq \sum_{q \leq Q}\left(\frac{2 x}{q} \log x+\frac{x}{\phi(q)}\right) \ll x(\log x)^{2} .
$$

(Throughout, the symbol "«", as in " $f(x) \ll g(x)$ " means "there exists a constant $c>0$ such that $f(x) \leq c g(x)$.") The Bombieri-Vinogradov theorem states that we can beat this trivial bound by an arbitrary power of $\log x$, provided $Q$ is a little smaller than $\sqrt{x}$ :

The Bombieri-Vinogradov theorem. For any given $A>0$ there exists a constant $B=B(A)$, such that

$$
\sum_{q \leq Q} \max _{\substack{a \bmod q \\(a, q)=1}}\left|\Theta(x ; q, a)-\frac{x}{\phi(q)}\right| \ll_{A} \frac{x}{(\log x)^{A}},
$$

where $Q=x^{1 / 2} /(\log x)^{B}$.

In fact one can take $B=2 A+5$, and one can also replace the summand here by the expression above with the maximum over $y$ as well as over $a$ (though we will not need to use this here).

3.2. Breaking the $x^{1 / 2}$-barrier. It is believed that estimates like that in the Bombieri-Vinogradov theorem hold with $Q$ significantly larger than $\sqrt{x}$; indeed Elliott and Halberstam conjectured [12] that one can take $Q=x^{c}$ for any constant $c<1$ :

The Elliott-Halberstam conjecture. For any given $A>0$ and $\eta, 0<\eta<\frac{1}{2}$, we have

$$
\sum_{q \leq Q} \max _{\substack{a \bmod q \\(a, q)=1}}\left|\Theta(x ; q, a)-\frac{x}{\phi(q)}\right| \ll \frac{x}{(\log x)^{A}},
$$

where $Q=x^{1 / 2+\eta}$.

However, it was shown in [19] that one cannot go so far as to take $Q=x /(\log x)^{B}$.

${ }^{11}$ Exceptional $q$ being those $q$ for which $\left|\Theta(x ; q, a)-\frac{x}{\phi(q)}\right|$ is not small, for some $a$ coprime to $q$. 
This conjecture was the starting point for the work of Goldston, Pintz, and Ylldirım 23] that was used by Zhang 68] (which we give in detail in the next section). It can be applied to obtain the following result, which we will prove.

Theorem 3.1 (Goldston, Pintz, and Ylldırım [23]). Let $k \geq 2, l \geq 1$ be integers, and let $0<\eta<1 / 2$ be such that

$$
1+2 \eta>\left(1+\frac{1}{2 l+1}\right)\left(1+\frac{2 l+1}{k}\right) .
$$

Assume that the Elliott-Halberstam conjecture holds with $Q=x^{1 / 2+\eta}$. If $x+a_{1}, \ldots, x+a_{k}$ is an admissible set of forms, then there are infinitely many integers $n$ such that at least two of $n+a_{1}, \ldots, n+a_{k}$ are prime numbers.

The conclusion here is exactly the statement of Zhang's main theorem.

If the Elliott-Halberstam conjecture conjecture holds for some $\eta>0$, then select $l$ to be an integer so large that $\left(1+\frac{1}{2 l+1}\right)<\sqrt{1+2 \eta}$. Theorem 3.1 then implies Zhang's theorem for $k=(2 l+1)^{2}$.

The Elliott-Halberstam conjecture seems to be too difficult to prove for now, but progress has been made when restricting to one particular residue class: Fix integer $a \neq 0$. We believe that for any fixed $\eta, 0<\eta<\frac{1}{2}$, one has

$$
\sum_{\substack{q \leq Q \\(q, a)=1}}\left|\Theta(x ; q, a)-\frac{x}{\phi(q)}\right| \ll \frac{x}{(\log x)^{A}},
$$

where $Q=x^{1 / 2+\eta}$, which follows from the Elliott-Halberstam conjecture (but is weaker).

The key to progress has been to notice that if one can "factor" the main variables here, then the extra flexibility allows one to make headway. For example, by factoring the modulus $q$ as, say, $d r$ where $d$ and $r$ are roughly some prespecified sizes. The simplest class of integers $q$ for which this can be done is the $y$-smooth integers, those integers whose prime factors are all $\leq y$. For example, if we are given a $y$-smooth integer $q$ and we want $q=d r$ with $d$ not much smaller than $D$, then we select $d$ to be the largest divisor of $q$ that is $\leq D$, and we see that $D / y<d \leq D$. This is precisely the class of moduli that Zhang considered.

The other "factorization" concerns the sum $\Theta(x ; q, a)$. The terms of this sum can be written as a sum of products, as we saw in (2.2); in fact we will decompose this further, partitioning the values of $a$ and $b$ (of (2.2) into different ranges.

Theorem 3.2 (Yitang Zhang's Theorem). There exist constants $\eta, \delta>0$ such that for any given integer $a$, we have

$$
\sum_{\substack{q \leq Q \\(q, a)=1 \\ q \text { is } y \text {-smooth } \\ q \text { square-free }}}\left|\Theta(x ; q, a)-\frac{x}{\phi(q)}\right| \ll_{A} \frac{x}{(\log x)^{A}},
$$

where $Q=x^{1 / 2+\eta}$ and $y=x^{\delta}$.

Zhang 68] proved his theorem for $\eta / 2=\delta=\frac{1}{1168}$, and his argument works provided $414 \eta+172 \delta<1$. We will prove this result, by a somewhat simpler proof, provided $162 \eta+90 \delta<1$, and the more sophisticated proof of [57] gives (3.5) provided $43 \eta+27 \delta<1$. We expect that this estimate holds for every $\eta \in[0,1 / 2)$ and 
every $\delta \in(0,1]$, but just proving it for any positive pair $\eta, \delta>0$ is an extraordinary breakthrough that has an enormous effect on number theory, since it is such an applicable result (and technique). This is the technical result that truly lies at the heart of Zhang's result about bounded gaps between primes, and sketching a proof of this is the focus of the second half of this article.

\section{The Goldston-Pintz-Yildirim ARgument}

The combinatorial argument of Goldston, Pintz, and Yıldırım [23] lies at the heart of the proof that there are bounded gaps between primes. (Henceforth we will call it "the GPY argument"; see 62] for a more complete discussion of their ideas.)

4.1. The setup. Let $\mathcal{H}=\left(a_{1}<a_{2}<\cdots<a_{k}\right)$ be an admissible $k$-tuple, and take $x>a_{k}$. Our goal is to select a weight for which weight $(n) \geq 0$ for all $n$, such that

$$
\sum_{x<n \leq 2 x} \operatorname{weight}(n)\left(\sum_{i=1}^{k} \theta\left(n+a_{i}\right)-\log 3 x\right)>0,
$$

where $\theta(m)=\log m$ if $m=p$ is prime, and $\theta(m)=0$ otherwise. If we can do this, then there must exist an integer $n$ such that

$$
\operatorname{weight}(n)\left(\sum_{i=1}^{k} \theta\left(n+a_{i}\right)-\log 3 x\right)>0 .
$$

In that case weight $(n) \neq 0$ so that weight $(n)>0$, and therefore

$$
\sum_{i=1}^{k} \theta\left(n+a_{i}\right)>\log 3 x \text {. }
$$

However, each $n+a_{i} \leq 2 x+a_{k}<2 x+x$, and so each $\theta\left(n+a_{i}\right)<\log 3 x$. This implies that at least two of the $\theta\left(n+a_{i}\right)$ are nonzero, that is, at least two of $n+a_{1}, \ldots, n+a_{k}$ are prime.

A simple idea, but the difficulty comes in selecting the function weight $(n)$ with these properties in such a way that we can evaluate the sums in (4.1). Moreover in 23. they also require that weight $(n)$ is sensitive to when each $n+a_{i}$ is "almost prime". All of these properties can be acquired by using a construction championed by Selberg. In order that weight $(n) \geq 0$, one can simply take it to be a square. Hence we select

$$
\operatorname{weight}(n):=\left(\sum_{\substack{d \mid \mathcal{P}(n) \\ d \leq R}} \lambda(d)\right)^{2},
$$

where the sum is over the positive integers $d$ that divide $\mathcal{P}(n)$, and

$$
\lambda(d):=\mu(d) G\left(\frac{\log d}{\log R}\right),
$$

where $G($.$) is a measurable, bounded function, supported only on [0,1]$, and $\mu$ is the Möbius function. Therefore $\lambda(d)$ is supported only on square-free, positive integers, that are $\leq R$. (This generalizes the discussion at the end of Section 2.4.) 
We can select $G(t)=(1-t)^{m} / m$ ! to obtain the results of this section, but it will benefit our understanding of the Maynard-Tao construction if we prove the GPY result for more general $G($.$) .$

4.2. Evaluating the sums over $n$. Expanding the above sum gives

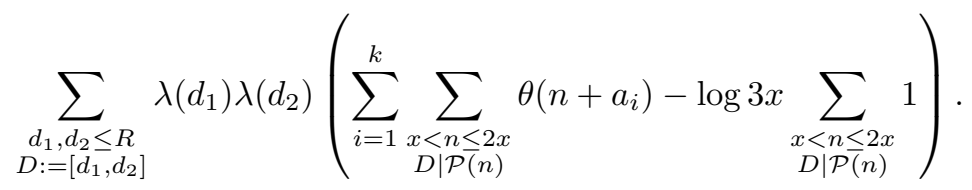

Let $\Omega(D)$ be the set of congruence classes $m(\bmod D)$ for which $D \mid P(m)$, and let $\Omega_{i}(D)$ be the set of congruence classes $m \in \Omega(D)$ with $\left(D, m+a_{i}\right)=1$. Hence, the parentheses in the above line equals

$$
\sum_{i=1}^{k} \sum_{m \in \Omega_{i}(D)} \sum_{\substack{x<n \leq 2 x \\ n \equiv m(\bmod D)}} \theta\left(n+a_{i}\right)-\log 3 x \sum_{m \in \Omega(D)} \sum_{\substack{x<n \leq 2 x \\ n \equiv m(\bmod D)}} 1,
$$

since $P(n) \equiv P(m)(\bmod D)$ whenever $n \equiv m(\bmod D)$.

Our first goal is to evaluate the sums over $n$. The final sum is easy; there are $x / D+O(1)$ integers in a given arithmetic progression with difference $D$, in an interval of length $x$. Here $D:=\left[d_{1}, d_{2}\right] \leq d_{1} d_{2} \leq R^{2}$, and so the error term here is much smaller than the main term if $R^{2}$ is much smaller than $x$. We will select $R \leq x^{\frac{1}{2}-o(1)}$ so that the sum of all of these error terms will be irrelevant to the subsequent calculations.

Counting the number of primes in a given arithmetic progression with difference $D$ in an interval of length $x$ is much more difficult. We expect that (3.1) holds so that each

$$
\Theta\left(2 x ; D, m+a_{i}\right)-\Theta\left(x ; D, m+a_{i}\right) \sim \frac{x}{\phi(D)} .
$$

The error terms here are larger and more care is needed. The sum of all of these error terms will be small enough to ignore, provided that the error terms are smaller than the main terms by an arbitrarily large power of $\log x$, at least on average. This shows why the Bombieri-Vinogradov theorem is so useful, since it implies the needed estimate provided $D<x^{1 / 2-o(1)}$ (which follows if $R<x^{1 / 4-o(1)}$ ). Going any further is difficult, so that the $\frac{1}{4}$ is an important barrier. Goldston, Pintz, and Yildırım showed that if one can go just beyond $\frac{1}{4}$, then one can prove that there are bounded gaps between primes, but there did not seem to be any techniques available to them to do so.

For the next part of this discussion we will ignore these accumulated error terms and estimate the size of the sum of the main terms. First, though, we need to better understand the sets $\Omega(D)$ and $\Omega_{i}(D)$. These sets may be constructed using the Chinese Remainder Theorem from the sets with $D$ prime. Therefore, if $\omega(D):=$ $|\Omega(D)|$, then $\omega($.$) is a multiplicative function. Moreover, each \left|\Omega_{i}(p)\right|=\omega(p)-$ 1 , which we denote by $\omega^{*}(p)$, and each $\left|\Omega_{i}(D)\right|=\omega^{*}(D)$, extending $\omega^{*}$ to be a multiplicative function. Putting this together, we obtain in (4.3) a main term of

$$
k \omega^{*}(D) \frac{x}{\phi(D)}-(\log 3 x) \omega(D) \frac{x}{D}=x\left(k \frac{\omega^{*}(D)}{\phi(D)}-(\log 3 x) \frac{\omega(D)}{D}\right) .
$$

This is typically negative, which explains why we cannot simply take the $\lambda(d)$ to all be positive in (4.2). Substituting this main term for (4.3) into each summand 
of (4.2), we obtain

$$
x\left(k \sum_{\substack{d_{1}, d_{2} \leq R \\ D:=\left[d_{1}, d_{2}\right]}} \lambda\left(d_{1}\right) \lambda\left(d_{2}\right) \frac{\omega^{*}(D)}{\phi(D)}-(\log 3 x) \sum_{\substack{d_{1}, d_{2} \leq R \\ D:=\left[d_{1}, d_{2}\right]}} \lambda\left(d_{1}\right) \lambda\left(d_{2}\right) \frac{\omega(D)}{D}\right) .
$$

The two sums over $d_{1}$ and $d_{2}$ in (4.4) are not easy to evaluate: The use of the Möbius function leads to many terms being positive, and many negative, so that there is a lot of cancelation. There are several techniques in analytic number theory that allow one to get accurate estimates for such sums, two more analytic ([23], [58]), the other more combinatorial ([62], [24]). We will discuss them all.

4.3. Evaluating the sums using Perron's formula. Perron's formula allows one to study inequalities using complex analysis:

$$
\frac{1}{2 i \pi} \int_{\operatorname{Re}(s)=2} \frac{y^{s}}{s} d s= \begin{cases}1 & \text { if } y>1 \\ 1 / 2 & \text { if } y=1 \\ 0 & \text { if } 0<y<1\end{cases}
$$

(Here the subscript " $\operatorname{Re}(s)=2$ " means that we integrate along the line $s: \operatorname{Re}(s)=$ 2 ; that is $s=2+i t$, as $t$ runs from $-\infty$ to $+\infty$.) So to determine whether $d<R$, we simply compute this integral with $y=R / d$. (The special case, $d=R$, has a negligible effect on our sums and can be avoided by selecting $R \notin \mathbb{Z}$.) Hence, the second sum in (4.4) equals

$$
\sum_{\substack{d_{1}, d_{2} \geq 1 \\ D:=\left[d_{1}, d_{2}\right]}} \lambda\left(d_{1}\right) \lambda\left(d_{2}\right) \frac{\omega(D)}{D} \cdot \frac{1}{2 i \pi} \int_{\operatorname{Re}\left(s_{1}\right)=2} \frac{\left(R / d_{1}\right)^{s_{1}}}{s_{1}} d s_{1} \cdot \frac{1}{2 i \pi} \int_{\operatorname{Re}\left(s_{2}\right)=2} \frac{\left(R / d_{2}\right)^{s_{2}}}{s_{2}} d s_{2} .
$$

Reorganizing this we obtain

$$
\frac{1}{(2 i \pi)^{2}} \int_{\substack{\operatorname{Re}\left(s_{1}\right)=2 \\ \operatorname{Re}\left(s_{2}\right)=2}}\left(\sum_{\substack{d_{1}, d_{2} \geq 1 \\ D:=\left[d_{1}, d_{2}\right]}} \frac{\lambda\left(d_{1}\right) \lambda\left(d_{2}\right)}{d_{1}^{s_{1}} d_{2}^{s_{2}}} \frac{\omega(D)}{D}\right) R^{s_{1}+s_{2}} \frac{d s_{2}}{s_{2}} \cdot \frac{d s_{1}}{s_{1}} .
$$

We will compute the sum in the middle in the special case that $\lambda(d)=\mu(d)$, the more general case following from a variant of this argument. Hence we have

$$
\sum_{d_{1}, d_{2} \geq 1} \frac{\mu\left(d_{1}\right) \mu\left(d_{2}\right)}{d_{1}^{s_{1}} d_{2}^{s_{2}}} \frac{\omega\left(\left[d_{1}, d_{2}\right]\right)}{\left[d_{1}, d_{2}\right]} .
$$

The summand is a multiplicative function, which means that we can evaluate it prime by prime. For any given prime $p$, the summand is 0 if $p^{2}$ divides $d_{1}$ or $d_{2}$ (since then $\mu\left(d_{1}\right)=0$ or $\mu\left(d_{2}\right)=0$ ). Therefore, we have only four cases to consider: $p \nmid d_{1}, d_{2} ; p\left|d_{1}, p \nmid d_{2} ; p \nmid d_{1}, p\right| d_{2} ; p\left|d_{1}, p\right| d_{2}$. So the $p$ th factor is

$$
1-\frac{1}{p^{s_{1}}} \cdot \frac{\omega(p)}{p}-\frac{1}{p^{s_{2}}} \cdot \frac{\omega(p)}{p}+\frac{1}{p^{s_{1}+s_{2}}} \cdot \frac{\omega(p)}{p} .
$$

We have seen that $\omega(p)=k$ for all sufficiently large $p$ so, in that case, the above becomes

$$
1-\frac{k}{p^{1+s_{1}}}-\frac{k}{p^{1+s_{2}}}+\frac{k}{p^{1+s_{1}+s_{2}}}
$$


In the analytic approach, we compare the integrand to a (carefully selected) power of the Riemann zeta function, which is defined as

$$
\zeta(s)=\sum_{n \geq 1} \frac{1}{n^{s}}=\prod_{p \text { prime }}\left(1-\frac{1}{p^{s}}\right)^{-1} \text { for } \operatorname{Re}(s)>1 .
$$

The $p$ th factor of $\zeta(s)$ is $\left(1-\frac{1}{p^{s}}\right)^{-1}$, so, as a first approximation, (4.7) is roughly

$$
\left(1-\frac{1}{p^{1+s_{1}+s_{2}}}\right)^{-k}\left(1-\frac{1}{p^{1+s_{1}}}\right)^{k}\left(1-\frac{1}{p^{1+s_{2}}}\right)^{k} .
$$

Substituting this back into (4.5), we obtain

$$
\frac{1}{(2 i \pi)^{2}} \iint_{\substack{\operatorname{Re}\left(s_{1}\right)=2 \\ \operatorname{Re}\left(s_{2}\right)=2}} \frac{\zeta\left(1+s_{1}+s_{2}\right)^{k}}{\zeta\left(1+s_{1}\right)^{k} \zeta\left(1+s_{2}\right)^{k}} G\left(s_{1}, s_{2}\right) \quad R^{s_{1}+s_{2}} \frac{d s_{2}}{s_{2}} \cdot \frac{d s_{1}}{s_{1}}
$$

where

$$
\begin{aligned}
G\left(s_{1}, s_{2}\right):= & \prod_{p \text { prime }}\left(1-\frac{1}{p^{1+s_{1}+s_{2}}}\right)^{k}\left(1-\frac{1}{p^{1+s_{1}}}\right)^{-k}\left(1-\frac{1}{p^{1+s_{2}}}\right)^{-k} \\
& \times\left(1-\frac{\omega(p)}{p^{1+s_{1}}}-\frac{\omega(p)}{p^{1+s_{2}}}+\frac{\omega(p)}{p^{1+s_{1}+s_{2}}}\right) .
\end{aligned}
$$

To determine the value of this integral, we move both contours in the integral slightly to the left of the lines $\operatorname{Re}\left(s_{1}\right)=\operatorname{Re}\left(s_{2}\right)=0$, and show that the main contribution comes, via Cauchy's theorem, from the pole at $s_{1}=s_{2}=0$. This can be achieved using our understanding of the Riemann zeta function and by noting that

$$
G(0,0):=\prod_{p \text { prime }}\left(1-\frac{\omega(p)}{p}\right)\left(1-\frac{1}{p}\right)^{-k}=C(a) \neq 0 .
$$

Remarkably, when one does the analogous calculation with the first sum in (4.4), one takes $k-1$ in place of $k$, and then

$$
G^{*}(0,0):=\prod_{p \text { prime }}\left(1-\frac{\omega^{*}(p)}{p-1}\right)\left(1-\frac{1}{p}\right)^{-(k-1)}=C(a),
$$

also. Since it is so unlikely that these two quite different products give the same constant by coincidence, one can feel sure that the method is correct!

This was the technique used in [23], and, although the outline of the method is quite compelling, the details of the contour shifting can be complicated.

4.4. Evaluating the sums using Fourier analysis. Both analytic approaches depend on the simple pole of the Riemann zeta function at $s=1$. The Fourier analytic approach (first used, to the author's knowledge, by Green and Tao, and in this context, on Tao's blog) avoids some of the more mysterious, geometric technicalities (which emerge when shifting contours in high-dimensional space), since the focus is more on the pole itself.

To appreciate the method, we prove a fairly general result, starting with smooth functions $F, H:[0,+\infty) \rightarrow \mathbb{R}$ that are supported on the finite interval $\left[0, \frac{\log R}{\log x}\right]$, and 
then we let $\lambda\left(d_{1}\right)=\mu\left(d_{1}\right) F\left(\frac{\log d_{1}}{\log x}\right)$ and $\lambda\left(d_{2}\right)=\mu\left(d_{2}\right) H\left(\frac{\log d_{2}}{\log x}\right)$. Note that $\lambda\left(d_{1}\right)$ is supported only when $d_{1} \leq R$, and similarly $\lambda\left(d_{2}\right)$. Our goal is to evaluate the sum

$$
\sum_{\substack{\left.d_{1}, d_{2} \geq 1 \\ D:=d_{1}, d_{2}\right]}} \mu\left(d_{1}\right) \mu\left(d_{2}\right) F\left(\frac{\log d_{1}}{\log x}\right) H\left(\frac{\log d_{2}}{\log x}\right) \frac{\omega(D)}{D} .
$$

The function $e^{t} F(t)$ (and similarly $e^{t} H(t)$ ) is also a smooth, finitely supported function. Such a function has a Fourier expansion

$$
e^{v} F(v)=\int_{t=-\infty}^{\infty} e^{-i t v} f(t) d t \quad \text { and so } F\left(\frac{\log d_{1}}{\log x}\right)=\int_{t=-\infty}^{\infty} \frac{f(t)}{d_{1}^{\frac{1+i t}{\log x}}} d t
$$

for some smooth, bounded function $f: \mathbb{R} \rightarrow \mathbb{C}$ that is rapidly decreasing 12 and therefore the tail of the integral (for instance when $|t|>\sqrt{\log x}$ ) does not contribute much. Substituting this and the analogous formula for $H($.$) into (4.8), we obtain$

$$
\int_{t, u=-\infty}^{\infty} f(t) h(u) \sum_{\substack{d_{1}, d_{2} \geq 1 \\ D:=\left[d_{1}, d_{2}\right]}} \frac{\mu\left(d_{1}\right) \mu\left(d_{2}\right)}{d_{1}^{\frac{1+i t}{\log x}} d_{2}^{\frac{1+i u}{\log x}}} \frac{\omega(D)}{D} d t d u
$$

We evaluated this same sum, (4.6) with $s_{1}=\frac{1+i t}{\log x}$ and $s_{2}=\frac{1+i u}{\log x}$, in the previous approach, and so we know that our integral equals

$$
\int_{t, u=-\infty}^{\infty} f(t) h(u) \frac{\zeta\left(1+\frac{2+i(t+u)}{\log x}\right)^{k}}{\zeta\left(1+\frac{1+i t}{\log x}\right)^{k} \zeta\left(1+\frac{1+i u}{\log x}\right)^{k}} G\left(\frac{1+i t}{\log x}, \frac{1+i u}{\log x}\right) d t d u .
$$

One can show that the contribution with $|t|,|u|>\sqrt{\log x}$ does not contribute much since $f$ and $h$ decay so rapidly. When $|t|,|u| \leq \sqrt{\log x}$, we are near the pole of $\zeta(s)$, and we can get very good approximations for the zeta values by using the Laurent expansion $\zeta(s)=1 / s+O(1)$. Moreover, $G\left(\frac{1+i t}{\log x}, \frac{1+i u}{\log x}\right)=G(0,0)+o(1)$ using its Taylor expansion, and therefore our integral is very close to

$$
\frac{G(0,0)}{(\log x)^{k}} \int_{t, u=-\infty}^{\infty} f(t) h(u) \frac{(1+i t)(1+i u)}{2+i(t+u)} d t d u .
$$

By the definition of $F$ we have $F^{\prime}(v)=-\int_{t=-\infty}^{\infty}(1+i t) e^{-(1+i t) v} f(t) d t$, and so

$$
\begin{aligned}
\int_{v=0}^{\infty} & F^{\prime}(v) H^{\prime}(v) d v \\
& =\int_{t, u=-\infty}^{\infty} f(t) h(u)(1+i t)(1+i u)\left(\int_{v=0}^{\infty} e^{-(1+i t) v-(1+i u) v} d v\right) d t d u \\
& =\int_{t, u=-\infty}^{\infty} f(t) h(u) \frac{(1+i t)(1+i u)}{2+i(t+u)} d t d u .
\end{aligned}
$$

Combining the last two displayed equations and remembering that $G(0,0)=C(a)$, we deduce that (4.8) is asymptotically equal to

$$
\frac{C(a)}{(\log x)^{k}} \int_{v=0}^{\infty} F^{\prime}(v) H^{\prime}(v) d v .
$$

One can do the analogous calculation with the first sum in (4.4), taking $k-1$ in place of $k$ and obtaining the constant $G^{*}(0,0)=C(a)$.

\footnotetext{
${ }^{12}$ That is, for any given $A>0$ there exists a constant $c_{A}$ such that $|f(\xi)| \leq c_{A}|\xi|^{-A}$.
} 
4.5. Evaluating the sums using Selberg's combinatorial approach, I. As discussed, the difficulty in evaluating the sums in (4.4) is that there are many positive terms and many negative terms. In developing his upper bound sieve method, Selberg encountered a similar problem and dealt with it in a surprising way, using combinatorial identities to remove this issue. The method rests on a reciprocity law: Suppose that $L(d)$ and $Y(r)$ are sequences of numbers, supported only on the square-free integers. If

$$
Y(r):=\mu(r) \sum_{m: r \mid m}^{\prime} L(m) \text { for all } r \geq 1
$$

then

$$
L(d)=\mu(d) \sum_{n: d \mid n}^{\prime} Y(n) \quad \text { for all } d \geq 1 .
$$

From here on, $\sum^{\prime}$ denotes the restriction to square-free integers that are $\leq R 13$

Let $\phi_{\omega}$ be the multiplicative function (defined here, only on square-free integers) for which $\phi_{\omega}(p)=p-\omega(p)$. We apply the above reciprocity law with

$$
L(d):=\frac{\lambda(d) \omega(d)}{d} \text { and } Y(r):=\frac{y(r) \omega(r)}{\phi_{\omega}(r)} .
$$

Now since $d_{1} d_{2}=D\left(d_{1}, d_{2}\right)$, we have

$$
\lambda\left(d_{1}\right) \lambda\left(d_{2}\right) \frac{\omega(D)}{D}=L\left(d_{1}\right) L\left(d_{2}\right) \frac{\left(d_{2}, d_{2}\right)}{\omega\left(\left(d_{2}, d_{2}\right)\right)}
$$

and therefore

$$
S_{1}:=\sum_{\substack{d_{1}, d_{2} \\ D:=\left[d_{1}, d_{2}\right]}}^{\prime} \lambda\left(d_{1}\right) \lambda\left(d_{2}\right) \frac{\omega(D)}{D}=\sum_{r, s} Y(r) Y(s) \sum_{\substack{d_{1}, d_{2} \\ d_{1}\left|r, d_{2}\right| s}}^{\prime} \mu\left(d_{1}\right) \mu\left(d_{2}\right) \frac{\left(d_{1}, d_{2}\right)}{\omega\left(\left(d_{1}, d_{2}\right)\right)} .
$$

The summand (of the inner sum) is multiplicative, and so we can work out its value, prime by prime. We see that if $p \mid r$ but $p \nmid s$ (or vice versa), then the sum is $1-1=0$. Hence if the sum is nonzero, then $r=s$ (as $r$ and $s$ are both square-free). In that case, if $p \mid r$, then the sum is $1-1-1+p / \omega(p)=\phi_{\omega}(p) / \omega(p)$. Hence the sum becomes

$$
S_{1}=\sum_{r} Y(r)^{2} \frac{\phi_{\omega}(r)}{\omega(r)}=\sum_{r} \frac{y(r)^{2} \omega(r)}{\phi_{\omega}(r)} .
$$

We will select

$$
y(r):=F\left(\frac{\log r}{\log R}\right)
$$

when $r$ is square-free, where $F(t)$ is measurable and supported only on $[0,1]$, and $y(r)=0$ otherwise. Hence, we now have a sum with all positive terms, so we do not have to fret about complicated cancelations.

\footnotetext{
${ }^{13}$ Selberg developed similar ideas in his construction of a small sieve, though he neither formulated a reciprocity law nor applied his ideas to the question of small gaps between primes.
} 
4.6. Sums of multiplicative functions. An important theme in analytic number theory is to understand the behavior of sums of multiplicative functions, some being easier than others. Multiplicative functions $f$, for which the $f(p)$ are fixed or almost fixed, were the first class of nontrivial sums to be determined. Indeed from the Selberg-Delange theorem 14 one can deduce that

$$
\sum_{n \leq x} \frac{g(n)}{n} \sim \kappa(g) \cdot \frac{(\log x)^{k}}{k !}
$$

where

$$
\kappa(g):=\prod_{p \text { prime }}\left(1+\frac{g(p)}{p}+\frac{g\left(p^{2}\right)}{p^{2}}+\cdots\right)\left(1-\frac{1}{p}\right)^{k}
$$

when $g(p)$ is typically "sufficiently close" to some given positive integer $k$ that the Euler product converges. Moreover, by partial summation, one deduces that

$$
\sum_{n \leq x} \frac{g(n)}{n} F\left(\frac{\log n}{\log x}\right) \sim \kappa(g)(\log x)^{k} \cdot \int_{0}^{1} F(t) \frac{t^{k-1}}{(k-1) !} d t .
$$

We apply this in the sum above, noting that here $\kappa(g)=1 / C(a)$, to obtain

$$
C(a) S_{1}=C(a) \sum_{r} \frac{\omega(r)}{\phi_{\omega}(r)} F\left(\frac{\log r}{\log R}\right)^{2} \sim(\log R)^{k} \cdot \int_{0}^{1} F(t)^{2} \frac{t^{k-1}}{(k-1) !} d t .
$$

A similar calculation reveals that

$$
C(a) \lambda(d) \sim \mu(d) \cdot\left(1-v_{d}\right)^{k} \int_{v_{d}}^{1} F(t) \frac{t^{k-1}}{(k-1) !} d t \cdot(\log R)^{k},
$$

where $v_{d}:=\frac{\log d}{\log R}$.

Remark 4.1. If, as in Section 4.4 we replace $\lambda\left(d_{1}\right) \lambda\left(d_{2}\right)$ by $\lambda_{1}\left(d_{1}\right) \lambda_{2}\left(d_{2}\right)$, then we obtain

$$
C(a) S_{1} \sim(\log R)^{k} \cdot \int_{0}^{1} F_{1}(t) F_{2}(t) \frac{t^{k-1}}{(k-1) !} d t .
$$

4.7. Selberg's combinatorial approach, II. A completely analogous calculation, but now applying the reciprocity law with

$$
L(d):=\frac{\lambda(d) \omega^{*}(d)}{\phi(d)} \text { and } Y(r):=\frac{y^{*}(r) \omega^{*}(r)}{\phi_{\omega}(r)}
$$

yields that

$$
S_{2}:=\sum_{\substack{d_{1}, d_{2} \\ D:=\left[d_{1}, d_{2}\right]}}^{\prime} \lambda\left(d_{1}\right) \lambda\left(d_{2}\right) \frac{\omega^{*}(D)}{\phi(D)}=\sum_{r} \frac{y^{*}(r)^{2} \omega^{*}(r)}{\phi_{\omega}(r)}
$$

\footnotetext{
${ }^{14}$ This also follows from the relatively easy proof of Theorem 1.1 of 40 .
} 
We need to determine $y^{*}(r)$ in terms of the $y(r)$, which we achieve by applying the reciprocity law twice:

$$
\begin{aligned}
y^{*}(r) & =\mu(r) \frac{\phi_{\omega}(r)}{\omega^{*}(r)} \sum_{d: r \mid d} \frac{\omega^{*}(d)}{\phi(d)} \mu(d) \frac{d}{\omega(d)} \sum_{n: d \mid n} \frac{y(n) \omega(n)}{\phi_{\omega}(n)} \\
& =\frac{r}{\phi(r)} \sum_{n: r \mid n} \frac{y(n)}{\phi_{\omega}(n / r)} \sum_{d: d / r \mid n / r} \mu(d / r) \frac{\omega^{*}(d / r) d / r}{\phi(d / r)} \omega(n / d) \\
& =r \sum_{n: r \mid n}^{\prime} \frac{y(n)}{\phi(n)}=\frac{r}{\phi(r)} \sum_{m:(m, r)=1}^{\prime} \frac{y(m r)}{\phi(m)} \\
& \sim \int_{\frac{\log r}{\log R}}^{1} F(t) d t \cdot \log R,
\end{aligned}
$$

where the last estimate was obtained by applying (4.11) with $k=1$, and taking care with the Euler product.

We now can insert this into (4.12) and apply (4.11) with $k$ replaced by $k-1$, noting that $\kappa\left(g^{*}\right)=1 / C(a)$, to obtain

$$
C(a) S_{2}=C(a) \sum_{r} \frac{y^{*}(r)^{2} \omega^{*}(r)}{\phi_{\omega}(r)} \sim(\log R)^{k+1} \cdot \int_{0}^{1}\left(\int_{t}^{1} F(u) d u\right)^{2} \frac{t^{k-2}}{(k-2) !} d t .
$$

Remark 4.2. If, as in Section 4.4 we replace $\lambda\left(d_{1}\right) \lambda\left(d_{2}\right)$ by $\lambda_{1}\left(d_{1}\right) \lambda_{2}\left(d_{2}\right)$, then we obtain

$$
C(a) S_{2} \sim(\log R)^{k+1} \cdot \int_{0}^{1}\left(\int_{t}^{1} F_{1}(u) d u\right)\left(\int_{t}^{1} F_{2}(v) d v\right) \frac{t^{k-2}}{(k-2) !} d t .
$$

4.8. Finding a positive difference: the proof of Theorem 3.1. From these estimates, we deduce that $C(a)$ times (4.4) is asymptotic to $x(\log 3 x)(\log R)^{k}$ times

$$
k \frac{\log R}{\log 3 x} \cdot \int_{0}^{1}\left(\int_{t}^{1} F(u) d u\right)^{2} \frac{t^{k-2}}{(k-2) !} d t-\int_{0}^{1} F(t)^{2} \frac{t^{k-1}}{(k-1) !} d t .
$$

Assume that the Elliott-Halberstam conjecture holds with exponent $\frac{1}{2}+\eta$, and let $R=\sqrt{Q}$. This then equals

$$
\int_{0}^{1} F(t)^{2} \frac{t^{k-1}}{(k-1) !} d t \cdot\left(\frac{1}{2}\left(\frac{1}{2}+\eta\right) \rho_{k}(F)-1\right)
$$

where

$$
\rho_{k}(F):=k \int_{0}^{1}\left(\int_{t}^{1} F(u) d u\right)^{2} \frac{t^{k-2}}{(k-2) !} d t / \int_{0}^{1} F(t)^{2} \frac{t^{k-1}}{(k-1) !} d t .
$$

Therefore if

$$
\frac{1}{2}\left(\frac{1}{2}+\eta\right) \rho_{k}(F)>1
$$

for some $F$ that satisfies the above hypotheses, then (4.13) is $>0$, which implies that (4.4) is $>0$, and so (4.1) is also $>0$, as desired.

We now need to select a suitable function $F(t)$ to proceed. A good choice is $F(t)=\frac{(1-t)^{\ell}}{\ell !}$. Using the beta integral identity

$$
\int_{0}^{1} \frac{v^{k}}{k !} \frac{(1-v)^{\ell}}{\ell !} d v=\frac{1}{(k+\ell+1) !}
$$


we obtain

$$
\int_{0}^{1} F(t)^{2} \frac{t^{k-1}}{(k-1) !} d t=\int_{0}^{1} \frac{(1-t)^{2 \ell}}{\ell !^{2}} \frac{t^{k-1}}{(k-1) !} d t=\frac{1}{(k+2 \ell) !}\left(\begin{array}{c}
2 \ell \\
\ell
\end{array}\right)
$$

and

$$
\begin{aligned}
\int_{0}^{1}\left(\int_{t}^{1} F(u) d u\right)^{2} \frac{t^{k-2}}{(k-2) !} d t & =\int_{0}^{1}\left(\frac{(1-t)^{\ell+1}}{\ell+1}\right)^{2} \frac{t^{k-2}}{(k-2) !} d t \\
& =\frac{1}{(k+2 \ell+1) !}\left(\begin{array}{c}
2 \ell+2 \\
\ell+1
\end{array}\right) .
\end{aligned}
$$

Therefore (4.14) is $>0$ if (3.4) holds, and so we deduce Theorem 3.1

To summarize: If the Elliott-Halberstam conjecture holds with exponent $\frac{1}{2}+\eta$ and if $\ell$ is an integer such that $1+2 \eta>\left(1+\frac{1}{2 \ell+1}\right)^{2}$, then for every admissible $k$-tuple, with $k=(2 \ell+1)^{2}$, there are infinitely many $n$ for which the $k$-tuple, evaluated at $n$, contains (at least) two primes.

\section{ZHANG'S MODIFICATIONS OF THE GPY ARGUMENT}

At the end of the previous section we saw that if the Elliott-Halberstam conjecture holds with any exponent $>\frac{1}{2}$, then for every admissible $k$-tuple (with $k$ sufficiently large), there are infinitely many $n$ for which the $k$-tuple contains two primes. However the Elliott-Halberstam conjecture remains unproven.

In (3.5) we stated Zhang's result, which breaks the $\sqrt{x}$-barrier (in such results), but at the cost of restricting the moduli to being $y$-smooth and restricting the arithmetic progressions $a(\bmod q)$ to having the same value of $a$ as we vary over $q$. Can the Goldston-Pintz-Yıldırım argument be modified to handle these restrictions?

5.1. Averaging over arithmetic progressions. In the GPY argument we need estimates for the number of primes in the arithmetic progressions $m+a_{i}(\bmod D)$ where $m \in \Omega_{i}(D)$. When using the Bombieri-Vinogradov theorem, it does not matter that $m+a_{i}$ varies as we vary over $D$, but it does matter when employing Zhang's Theorem 3.2 .

Zhang realized that one can exploit the structure of the sets $O_{i}(D)=\Omega_{i}(D)+a_{i}$, since they are constructed from the sets $O_{i}(p)$ for each prime $p$ dividing $D$ using the Chinese Remainder Theorem, to get around this issue:

Let $\nu(D)$ denote the number of prime factors of (square-free) $D$, so that $\tau(D)=$ $2^{\nu(D)}$. Any square-free $D$ can be written as $\left[d_{1}, d_{2}\right]$ for $3^{\nu(D)}$ pairs $d_{1}, d_{2}$, which means that we need an appropriate upper bound on

$$
\leq \sum_{D \leq Q}^{\prime} 3^{\nu(D)} \sum_{b \in O_{i}(D)}\left|\Theta(X ; D, b)-\frac{X}{\phi(D)}\right|
$$

where $Q=R^{2}$ and $X=x$ or $2 x$, for each $i$.

Let $L$ be the lcm of all of the $D$ in our sum. The set $O_{i}(L), \operatorname{reduced} \bmod D$, gives $\left|O_{i}(L)\right| /\left|O_{i}(D)\right|$ copies of $O_{i}(D)$, and so

$$
\frac{1}{\left|O_{i}(D)\right|} \sum_{b \in O_{i}(D)}\left|\Theta(X ; D, b)-\frac{X}{\phi(D)}\right|=\frac{1}{\left|O_{i}(L)\right|} \sum_{b \in O_{i}(L)}\left|\Theta(X ; D, b)-\frac{X}{\phi(D)}\right| .
$$


Now $\left|O_{i}(D)\right|=\omega^{*}(D) \leq(k-1)^{\nu(D)}$, and so $3^{\nu(D)}\left|O_{i}(D)\right| \leq \tau(D)^{A}$ for all squarefree $D$, where $A$ is chosen so that $2^{A}=3(k-1)$. The above is therefore

$$
\begin{aligned}
& \leq \sum_{D \leq Q}^{\prime} \tau(D)^{A} \cdot \frac{1}{\left|O_{i}(D)\right|} \sum_{b \in O_{i}(D)}\left|\Theta(X ; D, b)-\frac{X}{\phi(D)}\right| \\
& =\frac{1}{\left|O_{i}(L)\right|} \sum_{b \in O_{i}(L) D \leq Q} \sum_{D \leq Q}^{\prime} \tau(D)^{A} \cdot\left|\Theta(X ; D, b)-\frac{X}{\phi(D)}\right| \\
& \leq \max _{a \in \mathbb{Z}} \sum_{\substack{D \leq Q \\
(D, a)=1}}^{\prime} \tau(D)^{A} \cdot\left|\Theta(X ; D, a)-\frac{X}{\phi(D)}\right| .
\end{aligned}
$$

This can be bounded using Theorem [3.2, via a standard technical argument: By Cauchy's Theorem, the square of this is

$$
\leq \sum_{D \leq Q} \frac{\tau(D)^{2 A}}{D} \cdot \sum_{D \leq Q}^{\prime} D\left|\Theta(X ; D, b)-\frac{X}{\phi(D)}\right|^{2} .
$$

The first sum is $\leq \prod_{p \leq Q}\left(1+\frac{1}{p}\right)^{B}$ where $B=2^{2 A}$, which is $\leq(c \log Q)^{B}$ for some constant $c>0$, by Mertens's theorem. For the second sum, $D \Theta(X ; D, b) \leq$ $(X+D) \log X$, trivially, and $\frac{D}{\phi(D)} \leq \log X$ (again by Mertens's theorem), so the second sum is

$$
\leq 2 X \log X \sum_{D \leq Q}^{\prime}\left|\Theta(X ; D, b)-\frac{X}{\phi(D)}\right| .
$$

The sum in this equation may be bounded by Theorem 3.2 .

5.2. Restricting the support to smooth integers. Zhang simply took the same coefficients $y(r)$ as above, but now restricted to $x^{\delta}$-smooth integers, and he called this restricted class of coefficients, $z(r)$. Evidently the sum in (4.9) with $z(r)$ in place of $y(r)$ is bounded above by the sum in (4.9). The sum in (4.12) with $z(r)$ in place of $y(r)$ is a little more tricky, since we need a lower bound. Zhang proceeds by showing that if $L$ is sufficiently large and $\delta$ sufficiently small, then the two sums differ by only a negligible amount 15 In particular we will prove Zhang's theorem when

$$
162 \eta+90 \delta<1
$$

Zhang's argument to restrict the support to smooth integers, as just discussed in this subsection, holds when $\ell=431, k=(2 \ell+1)^{2}$, and $\eta=2 /(2 \ell+1)$.

\section{The GPY ARGUMENT IN HIGHER-DIMENSIONAL ANALYSIS}

In the GPY argument, we studied the divisors $d$ of the product of the $k$-tuple values; that is

$$
d \mid \mathcal{P}(n)=\left(n+a_{1}\right) \cdots\left(n+a_{k}\right)
$$

with $d \leq R$.

\footnotetext{
${ }^{15}$ Unbeknownst to Zhang, Motohashi and Pintz [50] had already given an argument to accomplish the goals of this section in the hope that someone might prove an estimate like (3.5)!
} 
Maynard and Tao (independently) realized that they could instead study the $k$-tuples of divisors $d_{1}, d_{2}, \ldots, d_{k}$ of each individual element of the $k$-tuple; that is

$$
d_{1}\left|n+a_{1}, d_{2}\right| n+a_{2}, \ldots, d_{k} \mid n+a_{k} .
$$

Now, instead of $d \leq R$, we take $d_{1} d_{2} \cdots d_{k} \leq R$.

6.1. The setup. One can proceed much as in the previous section, though technically it is easier to restrict our attention to when $n$ is in an appropriate congruence class $\bmod m$, where $m$ is the product of the primes for which $\omega(p)<k$, because if $\omega(p)=k$, then $p$ can only divide one $n+a_{i}$ at a time. Therefore we study

$$
\begin{aligned}
S_{0}:= & \sum_{r \in \Omega(m)} \sum_{\substack{n \sim x \\
n \equiv r(\bmod m)}}\left(\sum_{j=1}^{k} \theta\left(n+a_{j}\right)-h \log 3 x\right) \\
& \times\left(\sum_{d_{i} \mid n+a_{i} \text { for each } i} \lambda\left(d_{1}, \ldots, d_{k}\right)\right)^{2},
\end{aligned}
$$

which upon expanding, as $\left(d_{i}, m\right) \mid\left(n+a_{i}, m\right)=1$, equals

$$
\begin{gathered}
\sum_{\substack{d_{1}, \ldots, d_{k} \geq 1 \\
e_{1} \ldots, e_{k} \geq 1 \\
\left(d_{i} e_{i}, m\right)=1 \text { for each } i}} \lambda\left(d_{1}, \ldots, d_{k}\right) \lambda\left(e_{1}, \ldots, e_{k}\right) \\
\times \sum_{r \in \Omega(m)} \sum_{\substack{n \sim x \\
n \equiv r(\bmod m) \\
\left[d_{i}, e_{i}\right] \mid n+a_{i} \text { for each } i}}\left(\sum_{j=1}^{k} \theta\left(n+a_{j}\right)-h \log 3 x\right) .
\end{gathered}
$$

Next notice that $\left[d_{i}, e_{i}\right]$ is coprime with $\left[d_{j}, e_{j}\right]$ whenever $i \neq j$, since their gcd divides $\left(n+a_{j}\right)-\left(n+a_{i}\right)$, which divides $m$, and so equals 1 as $\left(d_{i} e_{i}, m\right)=1$. Hence, in our internal sum, the values of $n$ belong to an arithmetic progression with modulus $m \prod_{i}\left[d_{i}, e_{i}\right]$. Also notice that if $n+a_{j}$ is prime, then $d_{j}=e_{j}=1$.

Therefore, ignoring error terms,

$$
S_{0}=\sum_{1 \leq \ell \leq k} \frac{\omega(m)}{\phi(m)} S_{2, \ell} \cdot x-h \frac{\omega(m)}{m} S_{1} \cdot x \log 3 x,
$$

where

$$
S_{1}:=\sum_{\substack{d_{1}, \ldots, d_{k} \geq 1 \\ e_{1}, \ldots, e_{k} \geq 1 \\\left(d_{i}, e_{j}\right)=1 \text { for } i \neq j}} \frac{\lambda\left(d_{1}, \ldots, d_{k}\right) \lambda\left(e_{1}, \ldots, e_{k}\right)}{\prod_{i}\left[d_{i}, e_{i}\right]}
$$

and

$$
S_{2, \ell}:=\sum_{\begin{array}{c}
d_{1}, \ldots, d_{k} \geq 1 \\
e_{1}, \ldots, e_{k} \geq 1 \\
d_{i}, e_{j}=1 \text { for } \\
d_{\ell}=e_{\ell}=1
\end{array}} \frac{\lambda\left(d_{1}, \ldots, d_{k}\right) \lambda\left(e_{1}, \ldots, e_{k}\right)}{\prod_{i} \phi\left(\left[d_{i}, e_{i}\right]\right)} .
$$

These sums can be evaluated in several ways. Tao (see his blog) gave what is perhaps the simplest approach, generalizing the Fourier analysis technique discussed in Section 4.4 (see [58, Lemma 4.1]). Maynard [45] gave what is, to the author's taste, the more elegant approach, generalizing Selberg's combinatorial technique: 
6.2. The combinatorics. The reciprocity law generalizes quite beautifully to higher dimension: Suppose that $L(d)$ and $Y(r)$ are two sequences of complex numbers, indexed by $d, r \in \mathbb{Z}_{\geq 1}^{k}$, and nonzero only when each $d_{i}$ (or $r_{i}$ ) is square-free. Then

$$
L\left(d_{1}, \ldots, d_{k}\right)=\prod_{i=1}^{k} \mu\left(d_{i}\right) \sum_{\substack{r_{1}, \ldots, r_{k} \geq 1 \\ d_{i} \mid r_{i} \text { for all } i}} Y\left(r_{1}, \ldots, r_{k}\right)
$$

if and only if

$$
Y\left(r_{1}, \ldots, r_{k}\right)=\prod_{i=1}^{k} \mu\left(r_{i}\right) \sum_{\begin{array}{c}
d_{1}, \ldots, d_{k} \geq 1 \\
r_{i} \mid d_{i} \text { for all } i
\end{array}} L\left(d_{1}, \ldots, d_{k}\right) .
$$

We use this much as above, in the first instance with

$$
L\left(d_{1}, \ldots, d_{k}\right)=\frac{\lambda\left(d_{1}, \ldots, d_{k}\right)}{d_{1}, \ldots, d_{k}} \quad \text { and } \quad Y\left(r_{1}, \ldots, r_{k}\right)=\frac{y\left(r_{1}, \ldots, r_{k}\right)}{\phi_{k}\left(r_{1} \ldots r_{k}\right)},
$$

where

$$
y\left(r_{1}, \ldots, r_{k}\right)=F\left(\frac{\log r_{1}}{\log R}, \ldots, \frac{\log r_{k}}{\log R}\right)
$$

with $F \in \mathbb{C}\left[t_{1}, \ldots, t_{k}\right]$, such that that there is a uniform bound on all of the firstorder partial derivatives, and $F$ is only supported on

$$
T_{k}:=\left\{\left(t_{1}, \ldots, t_{k}\right): \text { each } t_{j} \geq 1, \text { and } t_{1}+\cdots+t_{k} \leq 1\right\} .
$$

Proceeding much as before, we obtain

$$
S_{1} \sim \sum_{r_{1}, \ldots, r_{k} \geq 1} \frac{y\left(r_{1}, \ldots, r_{k}\right)^{2}}{\phi_{k}\left(r_{1} \cdots r_{k}\right)} .
$$

6.3. Sums of multiplicative functions. By (4.10) we have

$$
\sum_{\substack{1 \leq n \leq N \\(n, m)=1}} \frac{\mu^{2}(n)}{\phi_{k}(n)}=\prod_{p \mid m} \frac{p-1}{p} \prod_{p \nmid m} \frac{(p-1) \phi_{k-1}(p)}{p \phi_{k}(p)} \cdot(\log N+O(1)) .
$$

We apply this $k$ times: first with $m$ replaced by $m r_{1} \cdots r_{k-1}$ and $n$ by $r_{k}$, then with $m$ replaced by $m r_{1} \cdots r_{k-2}$, etc. By the end we obtain

$$
C_{m}(a) \sum_{\substack{1 \leq r_{1} \leq R_{1}, 1 \leq r_{k} \leq R_{k}}} \frac{\mu^{2}\left(r_{1} \cdots r_{k} m\right)}{\phi_{k}\left(r_{1}, \ldots, r_{k}\right)}=\prod_{i}\left(\log R_{i}+O(1)\right),
$$

where

$$
C_{m}(a):=\prod_{p \mid m}\left(1-\frac{1}{p}\right)^{-k} \prod_{p \nmid m}\left(1-\frac{k}{p}\right)\left(1-\frac{1}{p}\right)^{-k} .
$$

From this and partial summation we deduce from (6.1) that

$$
C_{m}(a) S_{1} \sim(\log R)^{k} \cdot \int_{t_{1}, \ldots, t_{k} \in T_{k}} F\left(t_{1}, \ldots, t_{k}\right)^{2} d t_{k} \cdots d t_{1} .
$$


Had we stopped our calculation one step earlier, we would have found

$$
C_{m}(a) \sum_{\substack{1 \leq r_{1} \leq R_{1}, \ldots, \ldots \\ 1 \leq r_{k-1} \leq R_{k-1}}} \frac{\mu^{2}\left(r_{1} \cdots r_{k-1} m\right)}{\phi_{k}\left(r_{1}, \ldots, r_{k-1}\right)}=\frac{m}{\phi(m)} \cdot \prod_{i}\left(\log R_{i}+O(1)\right) .
$$

Remark 6.1. If we replace $\lambda(d) \lambda(e)$ by $\lambda_{1}(d) \lambda_{2}(e)$ in the definition of $S_{1}$, then the analogous argument yields $\int_{t \in T_{k}} F(t)^{2} d t$ replaced by $\int_{t \in T_{k}} F_{1}(t) F_{2}(t) d t$ in (6.4).

6.4. The combinatorics, II. We will deal only with the case $\ell=k$, the other cases being analogous. Now we use the higher-dimensional reciprocity law with

$$
L\left(d_{1}, \ldots, d_{k-1}\right)=\frac{\lambda\left(d_{1}, \ldots, d_{k-1}, 1\right)}{\phi\left(d_{1} \cdots d_{k-1}\right)} \text { and } Y_{k}\left(r_{1}, \ldots, r_{k-1}\right)=\frac{y_{k}\left(r_{1}, \ldots, r_{k-1}\right)}{\phi_{k}\left(r_{1} \cdots r_{k-1}\right)},
$$

where $d_{k}=r_{k}=1$, so that, with the exactly analogous calculations as before,

$$
S_{2, k} \sim \sum_{r_{1}, \ldots, r_{k-1} \geq 1} \frac{y_{k}\left(r_{1}, \ldots, r_{k-1}\right)^{2}}{\phi_{k}\left(r_{1} \cdots r_{k-1}\right)} .
$$

Using the reciprocity law twice to determine the $y_{k}(r)$ in terms of the $y(n)$, we obtain that

$$
y_{k}\left(r_{1}, \ldots, r_{k-1}\right) \sim \frac{\phi(m)}{m} \cdot \int_{t \geq 0} F\left(\rho_{1}, \ldots, \rho_{k-1}, t\right) d t \cdot \log R,
$$

where each $r_{i}=N^{\rho_{i}}$. Therefore, using (6.5), we obtain

$$
\begin{aligned}
C_{m}(a) S_{2, k} \sim & \int_{0 \leq t_{1}, \ldots, t_{k-1} \leq 1}\left(\int_{t_{k} \geq 0} F\left(t_{1}, \ldots, t_{k-1}, t_{k}\right) d t_{k}\right)^{2} d t_{k-1} \cdots d t_{1} \\
& \cdot \frac{\phi(m)}{m}(\log R)^{k+1}
\end{aligned}
$$

Remark 6.2. If we replace $\lambda(d) \lambda(e)$ by $\lambda_{1}(d) \lambda_{2}(e)$ in the definition of $S_{2}$, then the analogous argument yields $\left(\int_{t_{k}>0} F(t) d t_{k}\right)^{2}$ replaced by $\left(\int_{t_{k} \geq 0} F_{1}(t) d t_{k}\right)\left(\int_{t_{k} \geq 0} F_{2}(t) d t_{k}\right)$ in (6.6).

6.5. Finding a positive difference. By the Bombieri-Vinogradov theorem we can take $R=x^{1 / 4-o(1)}$, so that, by (6.4) and (6.6),

$$
\begin{aligned}
C_{m}(a) S_{0}= & \frac{\omega(m)}{m} x(\log 3 x)(\log R)^{k} \\
& \times\left(\frac{1}{4} \sum_{\ell=1}^{k} \int_{\substack{0 \leq t_{i} \leq 1 \text { for } \\
1 \leq i \leq k, \ell}}\left(\int_{t_{\ell} \geq 0} F\left(t_{1}, \ldots, t_{k}\right) d t_{\ell}\right)^{2} \prod_{\substack{1 \leq j \leq k \\
i \neq \ell}} d t_{j}\right. \\
& \left.-h \int_{t_{1}, \ldots, t_{k} \in T_{k}} F\left(t_{1}, \ldots, t_{k}\right)^{2} d t_{k} \cdots d t_{1}+o(1)\right) .
\end{aligned}
$$

One can show that the optimal choice for $F$ must be symmetric. Hence $S_{0}>0$ follows if there exists a symmetric $F$ (with the restrictions above) for which the ratio

satisfies $\rho(F)>4 h$.

$$
\rho(F):=\frac{k \int_{t_{1}, \ldots, t_{k-1} \geq 0}\left(\int_{t_{k} \geq 0} F\left(t_{1}, \ldots, t_{k}\right) d t_{k}\right)^{2} d t_{k-1} \cdots d t_{1}}{\int_{t_{1}, \ldots, t_{k} \geq 0} F\left(t_{1}, \ldots, t_{k}\right)^{2} d t_{k} \cdots d t_{1}}
$$


We have proved the following Proposition 6.3, which leaves us to find functions $F$ with certain properties in order to obtain the main results:

Proposition 6.3. Fix $h \geq 1$. Suppose that there exists $F \in \mathbb{C}\left(x_{1}, \ldots, x_{k}\right)$ which is measurable, is supported on $T_{k}$, for which there is a uniform bound on the first order partial derivatives, and such that $\rho(F)>4 h$. Then, for every admissible $k$-tuple of linear forms, there are infinitely many integers $n$ such that there are $>h$ primes amongst the $k$ linear forms when evaluated at $n$. If the Elliott-Halberstam conjecture holds, then we only need that $\rho(F)>2 h$.

6.6. A special case. If $F\left(t_{1}, \ldots, t_{k}\right)=f\left(t_{1}+\cdots+t_{k}\right)$, then since

$$
\int_{\substack{t_{1}, \ldots, t_{k} \geq 0 \\ t_{1}+\cdots+t_{k}=t}} d t_{k-1} \cdots d t_{1}=\frac{t^{k-1}}{(k-1) !}
$$

we deduce that

$$
\rho(F)=\rho_{k}(f)
$$

as defined in (4.14). That is, we have reverted to the original GPY argument, which was not quite powerful enough for our needs. We want to select $F$ that does not lead us back to the original GPY argument, so we should avoid selecting $F$ to be a function of one variable.

Since $F$ is symmetric, we can define the symmetric sums, $P_{j}=\sum_{i=1}^{k} t_{i}^{j}$. In the GPY argument $F$ was a function of $P_{1}$. A first guess might be to work now with functions of $P_{1}$ and $P_{2}$, so as to consider functions $F$ that do not appear in the GPY argument.

6.7. Maynard's $F \mathbf{s}$, and gaps between primes. For $k=5$, let

$$
F\left(t_{1}, \ldots, t_{5}\right)=70 P_{1} P_{2}-49 P_{1}^{2}-75 P_{2}+83 P_{1}-34 \text {. }
$$

A calculation yields that

$$
\rho(F)=\frac{1417255}{708216}>2 .
$$

Therefore, by Proposition 6.3, if we assume the Elliott-Halberstam conjecture with $h=1$, then for every admissible 5 -tuple of linear forms, there are infinitely many integers $n$ such that there are at least two primes amongst the five linear forms when evaluated at $n$. In particular, from the admissible forms $\{x, x+2, x+6, x+8, x+12\}$, we deduce that there are infinitely many pairs of distinct primes that differ by no more than 12. Also from the admissible forms $\{x+1,2 x+1,4 x+1,8 x+1,16 x+1\}$, we deduce that there are infinitely many pairs of distinct primes, $p, q$, for which $(p-1) /(q-1)=2^{j}$ for $j=0,1,2,3$, or 4 .

Maynard [45] showed that there exists a polynomial of the form

$$
\sum_{\substack{a, b \geq 0 \\ a+2 b \leq 11}} c_{a, b}\left(1-P_{1}\right)^{a} P_{2}^{b}
$$

with $k=105$, for which

$$
\rho(F)=4.0020697 \cdots .
$$

By Proposition 6.3 with $h=1$, we can then deduce that for every admissible 105tuple of linear forms, there are infinitely many integers $n$ such that there are at least two primes amongst the 105 linear forms when evaluated at $n$.

How did Maynard find his polynomial $F$ of the above form? The numerator and denominator of $\rho(F)$ are quadratic forms in the 42 variables $c_{a, b}$, say $v^{T} M_{2} v$ and 
$v^{T} M_{1} v$, respectively, where $v$ is the vector of $c$-values. The matrices $M_{1}$ and $M_{2}$ are easily determined. By the theory of Lagrangian multipliers, Maynard showed that

$$
M_{1}^{-1} M_{2} v=\rho(F) v,
$$

so that $\rho(F)$ is the largest eigenvalue of $M_{1}^{-1} M_{2}$, and $v$ is the corresponding eigenvector. These calculations are easily accomplished using a computer algebra package and yield the result above.

6.8. $F$ as a product of one-dimensional functions. We select

$$
F\left(t_{1}, \ldots t_{k}\right)= \begin{cases}g\left(k t_{1}\right) \cdots g\left(k t_{k}\right) & \text { if } t_{1}+\cdots+t_{k} \leq 1, \\ 0 & \text { otherwise }\end{cases}
$$

where $g$ is some integrable function supported only on $[0, T]$. Let $\gamma:=\int_{t>0} g(t)^{2} d t$, so that the denominator of $\rho(F)$ is

$$
\begin{aligned}
I_{k} & =\int_{t \in T_{k}} f\left(t_{1}, \ldots, t_{k}\right)^{2} d t_{k} \cdots d t_{1} \\
& \leq \int_{t_{1}, \ldots, t_{k} \geq 0}\left(g\left(k t_{1}\right) \cdots g\left(k t_{k}\right)\right)^{2} d t_{k} \cdots d t_{1}=k^{-k} \gamma^{k} .
\end{aligned}
$$

We rewrite the numerator of $\rho(F)$ as $L_{k}-M_{k}$ where

$$
\begin{aligned}
L_{k} & :=k \int_{t_{1}, \ldots, t_{k-1} \geq 0}\left(\int_{t_{k} \geq 0} g\left(k t_{1}\right) \cdots g\left(k t_{k}\right) d t_{k}\right)^{2} d t_{k-1} \cdots d t_{1} \\
& =k^{-k} \gamma^{k-1}\left(\int_{t \geq 0} g(t) d t\right)^{2} .
\end{aligned}
$$

As $g(t)$ is only supported in $[0, T]$, we have, by "Cauchying" and letting $u_{j}=k t_{j}$,

$$
\begin{aligned}
M_{k}: & =\int_{t_{1}, \ldots, t_{k-1} \geq 0}\left(\int_{t_{k} \geq 1-t_{1}-\cdots-t_{k-1}} g\left(k t_{1}\right) \cdots g\left(k t_{k}\right) d t_{k}\right)^{2} d t_{k-1} \cdots d t_{1} \\
& \leq k^{-k} T \int_{\substack{u_{1}, \ldots, u_{k} \geq 0 \\
u_{1}+\cdots+u_{k} \geq k}} g\left(u_{1}\right)^{2} \cdots g\left(u_{k}\right)^{2} d u_{1} \cdots d u_{k} .
\end{aligned}
$$

Now assume that $\mu:=\int_{t} t g(t)^{2} d t \leq(1-\eta) \int_{t} g(t)^{2} d t=(1-\eta) \gamma$ for some given $\eta>0$; that is, that the "weight" of $g^{2}$ is centered around values of $t \leq 1-\eta$. We have

$$
1 \leq \eta^{-2}\left(\frac{1}{k}\left(u_{1}+\cdots+u_{k}\right)-\mu / \gamma\right)^{2}
$$

whenever $u_{1}+\cdots+u_{k} \geq k$. Therefore,

$$
\begin{aligned}
M_{k} & \leq \eta^{-2} k^{-k} T \int_{u_{1}, \ldots, u_{k} \geq 0} g\left(u_{1}\right)^{2} \cdots g\left(u_{k}\right)^{2}\left(\frac{1}{k}\left(u_{1}+\cdots+u_{k}\right)-\mu / \gamma\right)^{2} d u_{1} \cdots d u_{k} \\
& =\eta^{-2} k^{-k-1} T \int_{u_{1}, \ldots, u_{k} \geq 0} g\left(u_{1}\right)^{2} \cdots g\left(u_{k}\right)^{2}\left(u_{1}^{2}-\mu^{2} / \gamma^{2}\right) d u_{1} \cdots d u_{k} \\
& =\eta^{-2} k^{-k-1} \gamma^{k-1} T\left(\int_{u \geq 0} u^{2} g(u)^{2} d u-\mu^{2} / \gamma\right) \\
& \leq \eta^{-2} k^{-k-1} \gamma^{k-1} T \int_{u \geq 0} u^{2} g(u)^{2} d u
\end{aligned}
$$


by symmetry. We deduce that

$$
\rho(F) \geq \frac{\left(\int_{t \geq 0} g(t) d t\right)^{2}-\frac{\eta^{-2} T}{k} \int_{u \geq 0} u^{2} g(u)^{2} d u}{\int_{t \geq 0} g(t)^{2} d t} .
$$

Notice that we can multiply $g$ through by a scalar and not affect the value in (6.7).

6.9. The optimal choice. We wish to find the value of $g$ that maximizes the right-hand side of (6.7). This can be viewed as an optimization problem:

$$
\begin{gathered}
\text { Maximize } \int_{t \geq 0} g(t) d t \text {, subject to the constraints } \\
\int_{t \geq 0} g(t)^{2} d t=\gamma \text { and } \int_{t \geq 0} t g(t)^{2} d t=\mu .
\end{gathered}
$$

One can approach this using the calculus of variations or even by discretizing $g$ and employing the technique of Lagrangian multipliers. The latter gives rise to (a discrete form of)

$$
\int_{t \geq 0} g(t) d t-\alpha\left(\int_{t \geq 0} g(t)^{2} d t-\gamma\right)-\beta\left(\int_{t \geq 0} t g(t)^{2} d t-\mu\right),
$$

for unknowns $\alpha$ and $\beta$. Differentiating with respect to $g(v)$ for each $v \in[0, T]$, we obtain

$$
1-2 \alpha g(v)-2 \beta v g(v)=0
$$

that is, after rescaling,

$$
g(t)=\frac{1}{1+A t} \text { for } 0 \leq t \leq T
$$

for some real $A>0$. We select $T$ so that $1+A T=e^{A}$, and let $A>1$. We then calculate the integrals in (6.7):

$$
\begin{aligned}
& \gamma=\int_{t} g(t)^{2} d t=\frac{1}{A}\left(1-e^{-A}\right), \\
& \int_{t} t g(t)^{2} d t=\frac{1}{A^{2}}\left(A-1+e^{-A}\right), \\
& \int_{t} t^{2} g(t)^{2} d t=\frac{1}{A^{3}}\left(e^{A}-2 A-e^{-A}\right), \\
& \text { and } \quad \int_{t} g(t) d t=1 \\
& \text { so that } \quad \eta=\frac{1-(A-1) e^{-A}}{A\left(1-e^{-A}\right)}>0 \text {, }
\end{aligned}
$$

which is necessary. Equation (6.7) then becomes

$$
\rho(F) \geq \frac{A}{\left(1-e^{-A}\right)}-\frac{e^{2 A}}{A k}\left(1-2 A e^{-A}-e^{-2 A}\right) \frac{\left(1-e^{-A}\right)^{2}}{\left(1-(A-1) e^{-A}\right)^{2}} \geq A-\frac{e^{2 A}}{A k} .
$$

Taking $A=\frac{1}{2} \log k+\frac{1}{2} \log \log k$, we deduce that

$$
\rho(F) \geq \frac{1}{2} \log k+\frac{1}{2} \log \log k-2 .
$$

Hence, for every $m \geq 1$, we find that $\rho(F)>4 m$ provided $e^{8 m+4}<k \log k$. 
This implies the following result:

Theorem 6.4. For any given integer $m \geq 2$, let $k$ be the smallest integer with $k \log k>e^{8 m+4}$. For any admissible $k$-tuple of linear forms $L_{1}, \ldots, L_{k}$ there exists infinitely many integers $n$ such that at least $m$ of the $L_{j}(n), 1 \leq j \leq k$, are prime.

For any $m \geq 1$, we let $k$ be the smallest integer with $k \log k>e^{8 m+4}$, so that $k>10000$; in this range it is known that $\pi(k) \leq \frac{k}{\log k-4}$. Next we let $x=2 k \log k>$ $10^{5}$, and for this range it is known that $\pi(x) \geq \frac{x}{\log x}\left(1+\frac{1}{\log x}\right)$. Hence

$$
\pi(2 k \log k)-\pi(k) \geq \frac{2 k \log k}{\log (2 k \log k)}\left(1+\frac{1}{\log (2 k \log k)}\right)-\frac{k}{\log k-4},
$$

and this is $>k$ for $k \geq 311$ by an easy calculation. We therefore apply the theorem with the $k$ smallest primes $>k$, which form an admissible set $\subset[1,2 k \log k]$, to obtain

Corollary 6.5. For any given integer $m \geq 2$, let $B_{m}=e^{8 m+5}$. There are infinitely many integers $x$ for which there are at least $m$ distinct primes within the interval $\left[x, x+B_{m}\right]$.

By a slight modification of this construction, Maynard in 45] obtains

$$
\rho(F) \geq \log k-2 \log \log k-1+o_{k \rightarrow \infty}(1)
$$

from which he analogously deduces that $B_{m} \ll m^{3} e^{4 m}$.

By employing Zhang's improvement, Theorem 3.2 to the Bombieri-Vinogradov theorem, one can improve this to $B_{m} \ll m e^{\left(4-\frac{28}{157}\right) m}$, and, if we assume the ElliottHalberstam conjecture, to $B_{m} \ll e^{(2+o(1)) m}$. The key to significantly improving these upper bounds on $B_{m}$ is to obtain a much better lower bound on $\rho(F)$.

6.10. Tao's upper bound on $\rho(F)$. By the Cauchy-Schwarz inequality we have

$$
\begin{aligned}
& \left(\int_{t_{k}=0}^{1-t_{1}-\cdots-t_{k-1}} F\left(t_{1}, \ldots, t_{k}\right) d t_{k}\right)^{2} \\
& \quad \leq \int_{t_{k}=0}^{1-t_{1}-\cdots-t_{k-1}} \frac{d t_{k}}{1-t_{1}-\cdots-t_{k-1}+(k-1) t_{k}} \\
& \quad \cdot \int_{t_{k}=0}^{1-t_{1}-\cdots-t_{k-1}} F\left(t_{1}, \ldots, t_{k}\right)^{2}\left(1-t_{1}-\cdots-t_{k-1}+(k-1) t_{k}\right) d t_{k} .
\end{aligned}
$$

Letting $u=A+(k-1) t$, we have

$$
\int_{0}^{A} \frac{d t}{A+(k-1) t}=\frac{1}{k-1} \int_{A}^{k A} \frac{d u}{u}=\frac{\log k}{k-1}
$$

Applying this with $A=1-t_{1}-\cdots-t_{k-1}$ and $t=t_{k}$ yields an upper bound for the numerator of $\rho(F)$ :

$$
\begin{aligned}
& \leq \frac{\log k}{k-1} \cdot \int_{t_{1}, \ldots, t_{k} \geq 0} F\left(t_{1}, \ldots, t_{k}\right)^{2} \sum_{j=1}^{k}\left(1-t_{1}-\cdots-t_{k}+k t_{j}\right) d t_{k} \cdots d t_{1} \\
& =\frac{k \log k}{k-1} \cdot \int_{t_{1}, \ldots, t_{k} \geq 0} F\left(t_{1}, \ldots, t_{k}\right)^{2} d t_{k} \cdots d t_{1} .
\end{aligned}
$$

Hence

$$
\rho(F) \leq \frac{k \log k}{k-1}=\log k+o_{k \rightarrow \infty}(1)
$$

so there is little room for improvement of Maynard's upper bound, (6.9). 


\section{Part 2. Primes in arithmetic progressions: breaking the $\sqrt{x}$-barrier}

Our goal, in the rest of the article, is to sketch the ideas behind a proof of Yitang Zhang's extraordinary result, given in (3.5), that primes are well distributed on average in the arithmetic progressions $a(\bmod q)$ with $q$ a little bigger than $\sqrt{x}$. We will see how this question fits into a more general framework, as developed by Bombieri, Friedlander, and Iwaniec [5], so that Zhang's results should also allow us to deduce analogous results for interesting arithmetic sequences other than the primes.

For the original, much deeper, and more complicated proof of Yitang Zhang, the reader is referred to the insightful exposition by Kowalski [42, which motivates and develops these difficult ideas with great clarity. One can also read a slightly different development of Zhang's theorem by Friedlander and Iwaniec [21, which incorporates various novel features.

To begin our discussion, we will introduce a key technique of analytic number theory: the idea of creating important sequences through convolutions.

\section{Convolutions in number theory}

The convolution of two functions $f$ and $g$, written $f * g$, is defined by

$$
(f * g)(n):=\sum_{a b=n} f(a) g(b),
$$

for every integer $n \geq 1$, where the sum is over all pairs of positive integers $a, b$ whose product is $n$. Hence if $\tau(n)$ counts the number of divisors of $n$, then

$$
\tau=1 * 1
$$

where 1 is the function with $1(n)=1$ for every $n \geq 1$. We already saw in (2.2) that if $L(n)=\log n$, then $\mu * L=\Lambda$. In the GPY argument we used that $(1 * \mu)(n)=0$ if $n>1$.

7.1. Dirichlet's divisor sum. There is no better way to understand why convolutions are useful than to present a famous argument of Dirichlet, estimating the average of $\tau(n)$ : If $n$ is square-free and has $k$ prime factors, then $\tau(n)=2^{k}$, so we see that $\tau(n)$ varies greatly depending on the arithmetic structure of $n$, but the average is more stable:

$$
\begin{aligned}
\frac{1}{x} \sum_{n \leq x} \tau(n) & =\frac{1}{x} \sum_{n \leq x} \sum_{d \mid n} 1=\frac{1}{x} \sum_{d \mid n} \sum_{\substack{n \leq x \\
d \mid n}} 1=\frac{1}{x} \sum_{d \leq x}\left[\frac{x}{d}\right] \\
& =\frac{1}{x} \sum_{d \leq x}\left(\frac{x}{d}+O(1)\right)=\sum_{d \leq x} \frac{1}{d}+O\left(\frac{1}{x} \sum_{d \leq x} 1\right) .
\end{aligned}
$$

One can approximate $\sum_{d \leq x} \frac{1}{d}$ by $\int_{1}^{x} d t / t=\log x$. Indeed the difference tends to a limit, the Euler-Mascheroni constant $\gamma:=\lim _{N \rightarrow \infty} \frac{1}{1}+\frac{1}{2}+\cdots+\frac{1}{N}-\log N$. Hence we have proved that the integers up to $x$ have $\log x+O(1)$ divisors, on average, which is quite remarkable for such a wildly fluctuating function.

Dirichlet studied this argument and noticed that when we approximate $[x / d]$ by $x / d+O(1)$ for large $d$, say for those $d$ in $(x / 2, x]$, then this is not really a very good approximation, since $\sum_{x / 2<d \leq x}(x / d+O(1))=O(x)$, a large cumulative error term. However we have the much more precise $[x / d]=1$ for each of these $d$, and so 
we can estimate this sum by $x / 2+O(1)$, a much better approximation. In general we write $n=d m$, where $d$ and $m$ are integers. When $d$ is small, then we should fix $d$ and count the number of such $m$, with $m \leq x / d$ (as we did above); but when $m$ is small, then we should fix $m$ and count the number of $d$ with $d \leq x / m$. In this way our sums are all over long intervals, which allows us to get an accurate approximation of their value:

$$
\begin{aligned}
\frac{1}{x} \sum_{n \leq x} \tau(n) & =\frac{1}{x} \sum_{n \leq x} \sum_{d m=n} 1=\frac{1}{x} \sum_{\substack { d \leq \sqrt{x} \\
\begin{subarray}{c}{n \leq x \\
d \mid n{ d \leq \sqrt { x } \\
\begin{subarray} { c } { n \leq x \\
d | n } }\end{subarray}} 1+\frac{1}{x} \sum_{\substack { m<\sqrt{x} \\
\begin{subarray}{c}{n \leq x \\
m \mid n{ m < \sqrt { x } \\
\begin{subarray} { c } { n \leq x \\
m | n } }\end{subarray}} 1-\frac{1}{x} \sum_{\substack{d \leq \sqrt{x} \\
m<\sqrt{x}}} 1 \\
& =\frac{1}{x} \sum_{d \leq \sqrt{x}}\left(\frac{x}{d}+O(1)\right)+\frac{1}{x} \sum_{\substack{m<\sqrt{x} \\
m}}\left(\frac{x}{m}+O(1)\right)-1+O\left(\frac{1}{\sqrt{x}}\right) \\
& =\log x+2 \gamma-1+O\left(\frac{1}{\sqrt{x}}\right),
\end{aligned}
$$

since $\sum_{n \leq N} 1 / n=\log N+\gamma+O(1 / N)$, an extraordinary improvement upon the earlier error term.

7.2. Vaughan's identity. If we sum (2.2) over all $n \leq x$ and trivially bound the contribution of the prime powers, then we obtain

$$
\Theta(x)=\sum_{a b \leq x} \mu(a) \log b+O(\sqrt{x}) .
$$

If we fix $a$ and then sum over $b \leq B$, where $B=[x / a]$, we obtain $\mu(a) \log B$ !, and we can approximate $B$ ! very well using Stirling's formula. Hence, the key difficulty in using this to approximate $\Theta(x)$ is to understand the sum of $\mu(a)$ times a smoothish function, for all integers $a \leq x$. We already discussed this a little in Section 2.3. and the problem remains that $\mu($.$) is not an easy function to sum.$

It is not difficult to find more complicated generalizations of our identity $\Lambda=$ $\mu * \log$, but to what end? Vinogradov made the extraordinary observation that, in certain ranges, it is possible to give good bounds on such convolutions, ignoring the precise details of the arithmetic function involved but rather getting bounds in terms of certain simpler sums involving the absolute value of those functions. The key is the bilinear shape of the convolutions. Vinogradov's strategy lies at the heart of all of the proofs in this area.

There are several suitable, more convoluted identities than (2.2), in which $\Lambda(n)$ is written as a linear combination of convolutions of arithmetic functions. The simplest is Vaughan's identity [65], and it will suffice for our needs.

$$
\text { Vaughan's identity: } \quad \Lambda_{\geq V}=\mu_{<U} * L-\mu_{<U} * \Lambda_{<V} * 1+\mu_{\geq U} * \Lambda_{\geq V} * 1,
$$

where $g_{>W}(n)=g(n)$ if $n>W$ and $g(n)=0$ otherwise; and $g=g_{\leq W}+g_{>W}$. To verify this identity, we manipulate the algebra of convolutions:

$$
\begin{aligned}
\Lambda_{\geq V} & =\Lambda-\Lambda_{<V}=(\mu * L)-\Lambda_{<V} *(1 * \mu) \\
& =\mu_{<U} * L+\mu_{\geq U} * L-\mu_{<U} * \Lambda_{<V} * 1-\mu_{\geq U} * \Lambda_{<V} * 1 \\
& =\mu_{<U} * L-\mu_{<U} * \Lambda_{<V} * 1+\mu_{\geq U} *\left(\Lambda * 1-\Lambda_{<V} * 1\right),
\end{aligned}
$$

from which we immediately deduce (7.1). 
The following identity, due to Heath-Brown [33], is used in [57] to get the strongest form of Zhang's theorem: If $n \leq U^{k}$, then

$$
\Lambda(n)=-\sum_{j=1}^{k}(-1)^{j}\left(\begin{array}{l}
k \\
j
\end{array}\right) \mu_{\leq U} * \mu_{\leq U} * \cdots * \mu_{\leq U} * \log * 1 * \cdots * 1(n),
$$

where $\mu_{\leq U}$ is convolved $j$ times and 1 is convolved $j-1$ times. This larger number of terms allows us to group divisors in such a way that we have closer control over their sizes.

\section{Distribution in ARITHMETIC Progressions}

8.1. General sequences in arithmetic progressions. One can ask whether any given sequence $(\beta(n))_{n \geq 1} \in \mathbb{C}$ is well distributed in arithmetic progressions modulo $q$. We begin by formulating an appropriate analogy to (3.2), which should imply nontrivial estimates in the range $q \leq(\log x)^{A}$ for any fixed $A>0$ : We say that $\beta$ satisfies a Siegel-Walfisz condition if, for any fixed $A>0$ and whenever $(a, q)=1$, we have

$$
\left|\sum_{\substack{n \leq x \\ n \equiv a(\bmod q)}} \beta(n)-\frac{1}{\phi(q)} \sum_{\substack{n \leq x \\(n, q)=1}} \beta(n)\right| \ll_{A} \frac{\|\beta\| x^{\frac{1}{2}}}{(\log x)^{A}},
$$

with $\|\beta\|=\|\beta\|_{2}$ where, as usual,

$$
\|\beta\|_{2}:=\left(\sum_{n \leq x}|\beta(n)|^{2}\right)^{\frac{1}{2}}
$$

Using Cauchy's inequality one can show that this assumption is "nontrivial" only for $q<(\log x)^{2 A}$; that is, when $x$ is very large compared to $q$.

Using the large sieve, Bombieri, Friedlander, and Iwaniec [5] proved two results that are surprisingly strong, given the weakness of the hypotheses. In the first they showed that if $\beta$ satisfies a Siegel-Walfisz condition 16 then it is well distributed for almost all arithmetic progressions $a(\bmod q)$, for almost all $q \leq x /(\log x)^{B}$ :

Theorem 8.1. Suppose that the sequence of complex numbers $\beta(n), n \leq x$, satisfies a Siegel-Walfisz condition. For any $A>0$ there exists $B=B(A)>0$ such that

$$
\sum_{q \leq Q} \sum_{a:(a, q)=1}\left|\sum_{n \equiv a(\bmod q)} \beta(n)-\frac{1}{\phi(q)} \sum_{(n, q)=1} \beta(n)\right|^{2} \ll\|\beta\|^{2} \frac{x}{(\log x)^{A}},
$$

where $Q=x /(\log x)^{B}$.

The analogous result for $\Lambda(n)$ is known as the Barban-Davenport-Halberstam theorem, and in that special case one can even obtain an asymptotic.

Before proceeding, let us assume, for the rest of this article, that we are given two sequences of complex numbers as follows:

- $\alpha(m), M<m \leq 2 M$, and $\beta(n), N<n \leq 2 N$, with $x^{1 / 3}<N \leq M \leq x^{2 / 3}$ and $M N \leq x$.

- $\beta(n)$ satisfies the Siegel-Walfisz condition.

${ }^{16}$ Their condition appears to be weaker than that assumed here, but it can be shown to be equivalent. 
- $\alpha(m) \ll \tau(m)^{A}(\log x)^{B}$ and $\beta(n) \ll \tau(n)^{A}(\log x)^{B}$ (these inequalities are satisfied by $\mu, 1, \Lambda, L$, and any convolutions of these sequences).

In their second result, Bombieri, Friedlander, and Iwaniec showed that rather general convolutions are well distributed ${ }^{17}$ for all arithmetic progressions $a(\bmod q)$, for almost all $q \leq x^{1 / 2} /(\log x)^{B}$.

Theorem 8.2. Suppose that $\alpha(m)$ and $\beta(n)$ are as above. For any $A>0$ there exists $B=B(A)>0$ such that

$$
\sum_{q \leq Q} \max _{a:(a, q)=1}\left|\sum_{n \equiv a(\bmod q)}(\alpha * \beta)(n)-\frac{1}{\phi(q)} \sum_{(n, q)=1}(\alpha * \beta)(n)\right| \ll\|\alpha\|\|\beta\| \frac{x^{1 / 2}}{(\log x)^{A}},
$$

where $Q=x^{1 / 2} /(\log x)^{B}$. Here we allow any $M$ and $N$ for which $M N \leq x$ and $x^{\epsilon} \ll M, N \ll x^{1-\epsilon}$.

The ranges for $M$ and $N$ are quite restricted in the result as we have stated it, though the proof is valid in the wider range, $N \geq \exp \left((\log x)^{\epsilon}\right)$ and $M \geq$ $(\log x)^{2 B+4}$. However this is still not a wide enough range to deduce the BombieriVinogradov theorem for primes from (2.2), but it is wide enough if one uses Vaughan's identity (7.1). This proof of the Bombieri-Vinogradov theorem follows the pattern laid down by Vinogradov, in that it seems to be less dependent on specific properties of the primes.

The restriction on the moduli staying below $x^{1 / 2}$ has been a major barrier to the development of analytic number theory 18

In the Bombieri-Vinogradov theorem one bounds the largest error term in the prime number theorem as one varies over all arithmetic progressions mod $q$, averaging over $q \leq Q$. In some applications (such as the one here), it suffices to bound the error term in the prime number theorem for the arithmetic progression $a(\bmod q)$, averaging over $q$, for one given integer $a$, as long as we obtain the same bound for all integers $a$.

Bombieri, Friedlander, and Iwaniec [5] made the following conjecture 19

Conjecture 8.3 (Generalized Elliott-Halberstam Conjecture). Suppose that $\alpha(\mathrm{m})$ and $\beta(n)$ are as above. For any $A, \epsilon>0$, we have

$$
\sum_{\substack{q \leq Q \\(q, a)=1}}\left|\sum_{n \equiv a(\bmod q)}(\alpha * \beta)(n)-\frac{1}{\phi(q)} \sum_{(n, q)=1}(\alpha * \beta)(n)\right| \ll\|\alpha\|\|\beta\| \frac{x^{1 / 2}}{(\log x)^{A}},
$$

where $Q=x^{1-\epsilon}$, for every integer a.

It is shown in 58] (by further developing the ideas described in this article) that if we assume this generalized Elliott-Halberstam conjecture then for every admissible 3-tuple of linear forms, there are infinitely many integers $n$ such that there are at least two primes amongst the three linear forms when evaluated at $n$.

\footnotetext{
${ }^{17}$ This possibility has its roots in papers of Gallagher [22] and of Motohashi [49].

${ }^{18}$ There had been some partial progress with moduli $>x^{1 / 2}$, as in [6], but no upper bounds that "win" by an arbitrary power of $\log x$ (which is what is essential to many applications).

${ }^{19}$ They actually conjectured that one can take $Q=x /(\log x)^{B}$. They also conjectured that if one assumes the Siegel-Walfisz condition with $\|\beta\|_{s} N^{1-\frac{1}{s}}$ in place of $\|\beta\| N^{\frac{1}{2}}$, then we may replace $\|\alpha\|\|\beta\| x^{1 / 2}$ in the upper bound here by $\|\alpha\| M^{1-\frac{1}{r}}\|\beta\| N^{1-\frac{1}{s}}$.
} 
In particular, from the admissible forms $\{x, x+2, x+6\}$, we deduce that there are infinitely many pairs of distinct primes that differ by no more than 6 .

The extraordinary work of Zhang breaks through the $\sqrt{x}$ barrier in some generality, working with moduli slightly larger than $x^{1 / 2}$, though his moduli are $y$-smooth, with $y=x^{\delta}$. The key result is as follows.

Theorem 8.4. Suppose that $\alpha(m)$ and $\beta(n)$ are as above. There exist constants $\eta, \delta>0$ such that, for any $A>0$,

$$
\sum_{\substack{q \leq Q \\ P(q) \leq x \\(q, a)=1 \\ q \text { square-free }}}\left|\sum_{\substack{n \leq x \\ n \equiv a(\bmod q)}}(\alpha * \beta)(n)-\frac{1}{\phi(q)} \sum_{\substack{n \leq x \\(n, q)=1}}(\alpha * \beta)(n)\right| \ll_{A}\|\alpha\|\|\beta\| \frac{x^{1 / 2}}{(\log x)^{A}},
$$

where $Q=x^{1 / 2+\eta}$ for any integer $a$.

One then can deduce the same result with the support for $\alpha$ and $\beta$ expanded to the wider range

$$
x^{1 / 3}<m, n \leq x^{2 / 3} \text { with } m n \leq x .
$$

One proves this by carefully dissecting this range up into dyadic ranges (that is, of the form $M<m \leq 2 M$ and $N<n \leq 2 N$ ) as well as possible and then carefully accounting for any $(m, n)$ pairs missed.

8.2. The deduction of the main theorem for primes. We will bound each term that arises from Vaughan's identity (17.1) with $U=V=x^{1 / 3}$, rewritten as

$$
\begin{aligned}
\Lambda= & \Lambda_{<x^{1 / 3}}+\mu_{<x^{1 / 3}} * L-(\mu * \Lambda)_{<x^{1 / 3}} * 1_{\geq x^{2 / 3}} \\
& -\mu_{<x^{1 / 3}} * \Lambda_{<x^{1 / 3}} * 1_{<x^{2 / 3}}+\mu_{\geq x^{1 / 3}} * \Lambda_{\geq x^{1 / 3}} * 1 .
\end{aligned}
$$

The first term is acceptably small, simply by taking absolute values. For the second term we write $\left(\mu_{<x^{1 / 3}} * L\right)(n)=\sum_{u m=n, u<x^{1 / 3}} \mu(u) \log m$, to obtain the difference

$$
\sum_{\substack{u<x^{1 / 3} \\(u, q)=1}} \mu(u)\left(\sum_{\substack{x / u<m \leq 2 x / u \\ m \equiv a / u(\bmod q)}} \log m-\frac{1}{\phi(q)} \sum_{\substack{x / u<m \leq 2 x / u \\(m, q)=1}} \log m\right) .
$$

Writing $M=x / u$, the inner sum is the difference between the sum of $\log m$ in $(M, 2 M]$ over an arithmetic progression $b(\bmod q)$ with $(b, q)=1$, minus the average of such sums. Now if $n_{-}=[M / q]$ and $n_{+}=[2 M / q]$, then, since $\log q[m / q]<$ $\log m<\log q([m / q]+1)$, such a sum is greater than $\sum_{n_{-}<n<n_{+}-1} \log q n$ and is less than $\sum_{n_{+} \leq n \leq n_{+}-1} \log q n$. The difference between these bounds is $\ll \log M$, and hence this is our bound on the term in parentheses. Summing over $u$ yields a bound that is acceptably small.

We deal with the third term, by the same argument as just above, since we obtain an inner sum of 1 , over the values of $m$ in an interval of an arithmetic progression, and then we obtain a bound that is acceptably small. 
We are left to work with two sums of convolutions:

$$
\begin{gathered}
\sum_{\substack{m n \asymp x \\
m n \equiv a(\bmod q)}}\left(\mu_{<x^{1 / 3}} * \Lambda_{<x^{1 / 3}}\right)(m) 1_{<x^{2 / 3}}(n) \text { and } \\
\sum_{\substack{m n \asymp x \\
m n \equiv a(\bmod q)}}\left(\Lambda_{\geq x^{1 / 3}} * 1\right)(m) \mu_{\geq x^{1 / 3}}(n),
\end{gathered}
$$

where $x^{1 / 3} \ll m, n \ll x^{2 / 3}$, and each convolution takes the form $\alpha(m) \beta(n)$ with $\alpha(m)$ and $\beta(n)$ as above. The result then follows from Zhang's result, as discussed at the end of the last subsection.

8.3. Further reductions. We reduce Theorem 8.4 further. The first observation is that we can restrict our moduli to those with $<C \log \log x$ prime factors, for some large $C>0$, since the moduli with more prime factors are rare and thus contribute little to the sum. Since the moduli are $y$-smooth, they can be factored as $q r$ where $N /\left(y x^{\epsilon}\right)<r \leq N / x^{\epsilon}$; moreover, as the modulus does not have a lot of prime factors, one can select $q$ and $r$ so that the smallest prime factor of $q$, denoted $p(q)$, is $\geq D_{0}:=x^{\epsilon / \log \log x}$. Hence we may also now assume

- $r \in(R, 2 R]$ with $P(r) \leq y$ with $y:=x^{\delta}$.

- $q \in(Q, 2 Q]$ with $D_{0}<p(q) \leq P(q) \leq y$.

- $N /\left(y x^{\epsilon}\right)<R \leq N / x^{\epsilon}$ and $x^{1 / 2} /(\log x)^{B}<Q R \leq x^{1 / 2+\eta}$.

In [57, some gains are made by working instead with the full set of moduli that have this kind of convenient factorization, rather than restricting attention just to those moduli which are $y$-smooth.

We begin by noting that

$$
\begin{aligned}
& \sum_{n \equiv a(\bmod q r)} \gamma(n)-\frac{1}{\phi(q r)} \sum_{\substack{(n, q r)=1 \\
n \equiv a(\bmod q r)}} \gamma(n) \\
& =\sum_{\substack{(n) \\
n \equiv a(\bmod r)}} \gamma(n) \\
& +\frac{1}{\phi(q)}\left(\sum_{\substack{(n, q)=1 \\
n \equiv a(\bmod r)}} \gamma(n)-\frac{1}{\phi(r)} \sum_{\substack{(n, q)=1 \\
(n, r)=1}} \gamma(n)\right)
\end{aligned}
$$

with $\gamma=\alpha * \beta$. We sum the absolute value of these terms, over the moduli $d \in[D, 2 D]$, factored into $q r$ as above. Since $\beta(n)$ satisfies the Siegel-Walfisz criterion, we can deduce that $\beta(n) 1_{(n, q)=1}$ also satisfies it, and therefore Theorem 8.2 is applicable for $\alpha(m) * \beta(n) 1_{(n, q)=1}$; this allows us to bound the sum of the second terms here, suitably. Hence it remains to prove

$$
\begin{aligned}
& \sum_{\substack{q \in[Q, 2 Q] \\
D_{0}<p(q) \leq P(q) \leq y}} \sum_{\substack{r \in[R, 2 R], P(r) \leq y \\
q r \\
\text { square-free }}}\left|\sum_{\substack{n \equiv a(\bmod r) \\
n \equiv b(\bmod q)}}(\alpha * \beta)(n)-\sum_{\substack{n \equiv a(\bmod r) \\
n \equiv b^{\prime}(\bmod q)}}(\alpha * \beta)(n)\right| \\
& \ll_{A}\|\alpha\|\|\beta\| \frac{x^{1 / 2}}{(\log x)^{A}},
\end{aligned}
$$

for any integers $a, b, b^{\prime}$ with $p\left(a b b^{\prime}\right)>y$. 


\section{Removing the Weights, AND AN UNWEIGHTED ARITHMETIC PROGRESSION}

At first sight it seems unlikely that one can estimate (8.1) since it involves unspecified sequences $\alpha$ and $\beta$. However we will use the Cauchy-Schwarz inequality to obtain an upper bound which depends only on the mean squares of $\alpha$ and $\beta$.

9.1. Removing the weights. In this section we use Cauchy's inequality to "unfold" (8.1), so as to remove the $\alpha$ and $\beta$ from the innermost sum. Surprisingly, this simple procedure can be applied so as to avoid much loss.

In the left-hand side of (8.1) we replace the absolute value in the $(q, r)$ term by a complex number $c_{q, r}$ of absolute value 1, to obtain, after a little rearranging,

$$
\sum_{r} \sum_{m} \alpha(m)\left(\sum_{q} \sum_{n: m n \equiv a(\bmod r)} c_{q, r} \beta(n)\left(1_{m n \equiv b(\bmod q)}-1_{m n \equiv b^{\prime}(\bmod q)}\right)\right) .
$$

By the Cauchy-Schwarz inequality the square of this is

$$
\leq \sum_{r} \sum_{m}|\alpha(m)|^{2} \leq R\|\alpha\|^{2}
$$

times

$$
\sum_{r} \sum_{m}\left|\sum_{q} \sum_{n: m n \equiv a(\bmod r)} c_{q, r} \beta(n)\left(1_{m n \equiv b(\bmod q)}-1_{m n \equiv b^{\prime}(\bmod q)}\right)\right|^{2} .
$$

When we expand the square, we obtain the sum of four terms of the form

$$
\begin{aligned}
\pm \sum_{r} & \sum_{m} \sum_{q_{1}, q_{2}} \sum_{\substack{n_{1}, n_{2} \\
m n_{1} \equiv m n_{2} \equiv a(\bmod r)}} c_{q_{1}, r} \overline{c_{q_{2}, r}} \\
& \times \beta\left(n_{1}\right) \overline{\beta\left(n_{2}\right)} 1_{m n_{1} \equiv b_{1}\left(\bmod q_{1}\right)} 1_{m n_{2} \equiv b_{2}\left(\bmod q_{2}\right)} \\
= & \pm \sum_{r} \sum_{q_{1}, q_{2}} \sum_{\substack{n_{1}, n_{2} \\
n_{1} \equiv n_{2}(\bmod r)}} c_{q_{1}, r} \overline{c_{q_{2}, r}} \beta\left(n_{1}\right) \overline{\beta\left(n_{2}\right)} \cdot \sum_{m} \begin{array}{c}
1_{m \equiv b_{1} / n_{1}\left(\bmod q_{1}\right)} \equiv b_{2} / n_{2}\left(\bmod q_{2}\right) \\
m \equiv a / n_{1}(\bmod r)
\end{array}
\end{aligned}
$$

where we get "+" when $b_{1}=b_{2}=b$ or $b^{\prime}$ and "-" otherwise, since $(m n, q r)=1$.

We have achieved our goal of having an unweighted innermost sum. Indeed, if it is nonzero 20 then it is just the number of integers in an interval of an arithmetic progression with common difference $r\left[q_{1}, q_{2}\right]$.

9.2. The main terms. The number of integers in an interval of length $M$ from an arithmetic progression with common difference $r\left[q_{1}, q_{2}\right]$ is

$$
\frac{M}{r\left[q_{1}, q_{2}\right]}+O(1)
$$

\footnotetext{
${ }^{20}$ If the congruences are incompatible, then this sum cannot possibly contain any integers, and so is 0 . Since $\left(r, q_{1} q_{2}\right)=1$, they are compatible unless $b_{1} / n_{1} \not \equiv b_{2} / n_{2}\left(\bmod \left(q_{1}, q_{2}\right)\right)$. Note that this criterion is irrelevant if $\left(q_{1}, q_{2}\right)=1$.
} 
We study now the sum of the "main terms", the $M / r\left[q_{1}, q_{2}\right]$. First, for the terms with $\left(q_{1}, q_{2}\right)=1$ the main terms sum to

$$
\pm \sum_{r} \sum_{\substack{q_{1}, q_{2} \\\left(q_{1}, q_{2}\right)=1}} \sum_{\substack{n_{1}, n_{2} \\ n_{1} \equiv n_{2}(\bmod r)}} c_{q_{1}, r} \overline{c_{q_{2}, r}} \beta\left(n_{1}\right) \overline{\beta\left(n_{2}\right)} \cdot \frac{M}{r q_{1} q_{2}},
$$

which is independent of the values of $b_{1}, b_{2}$ and hence they cancel when we sum over the four terms (and the two "+", and two "-", signs). For the terms with $\left(q_{1}, q_{2}\right) \neq$ 1 , we have $\left(q_{1}, q_{2}\right) \geq D_{0}$ (since the prime factors of the $q_{i}$ are all $\left.\geq D_{0}\right)$, and it is not difficult to show that these are $\ll x(\log x)^{O(1)} / R D_{0}$, which is acceptably small.

9.3. The error terms and the advent of exponential sums. The $O(1)$ 's in (9.2) can add up to a total that is far too large. One can show that in most of the terms of the sum, the common difference of the arithmetic progression is larger than the length of the interval, so the correct count is either 0 or 1 : it is hardly surprising that an error term of $O(1)$ is too insensitive to help us.

To proceed, instead of approximating, we will give a precise formula for the number of integers in an arithmetic progression in an interval, using a sum of exponentials. By the Chinese Remainder Theorem, we can rewrite our triple of congruence conditions

$$
m \equiv b_{1} / n_{1}\left(\bmod q_{1}\right), m \equiv b_{2} / n_{2}\left(\bmod q_{2}\right), m \equiv a / n_{1}(\bmod r)
$$

as one,

$$
m \equiv m_{0}\left(n_{1}, n_{2}\right)(\bmod q),
$$

where $q=r g \ell_{1} \ell_{2}$, when there is a solution, which happens if and only if $b_{1} / n_{1} \equiv$ $b_{2} / n_{2}(\bmod g)$, where $g=\left(q_{1}, q_{2}\right)$, and we now define $\ell_{1}=q_{1} / g, \ell_{2}=q_{2} / g$.

To identify whether $m$ is in a given interval $I$, we use Fourier analysis. We define $e(t):=e^{2 i \pi t}$ for any real number $t$, and then $e_{q}(t)=e(t / q)$. The discrete Fourier transform is defined by

$$
\hat{f}(h):=\sum_{b(\bmod q)} f(b) e_{q}(h b),
$$

for any function $f$ of period $q$. If $f$ is any such function and $I($.$) is the characteristic$ function for the interval $(M, 2 M]$, then

$$
\sum_{m \in I} f(m)=\frac{1}{q} \sum_{h(\bmod q)} \hat{I}(h) \hat{f}(-h)
$$

is an example of Plancherel's formula. This has a "main term" at $h=0$ (which is the same as the main term we found above, in that special case). The coefficients $\hat{I}(h)$ are easily evaluated and bounded:

$$
\hat{I}(h)=\sum_{m=M+1}^{2 M} e_{q}(h m)=e_{q}(2 h M) \cdot \frac{e_{q}(h M)-1}{e_{q}(h)-1} .
$$

The numerator has absolute value $\leq 2$, and, using the Taylor expansion, the denominator has absolute value $\asymp|h| / q$. Hence

$$
|\hat{I}(h)| \ll \min \{M, q /|h|\} .
$$


We apply (9.3) with $f=\sum_{i} c_{i} 1_{m \equiv a_{i}(\bmod q)}$, take absolute values, and use our bounds for $|\hat{I}(h)|$, to obtain

$$
\left|\sum_{i} c_{i}\left(\sum_{\substack{m \asymp M \\ m \equiv a_{i}(\bmod q)}} 1-\frac{M}{q}\right)\right| \ll \sum_{\substack{0 \leq j \leq J \\ H_{j}:=2^{j} q / M}} \frac{1}{H_{j}} \sum_{1 \leq|h| \leq H_{j}}\left|\sum_{i} c_{i} e_{q}\left(a_{i} h\right)\right| .
$$

The error terms in (9.2) are bounded by

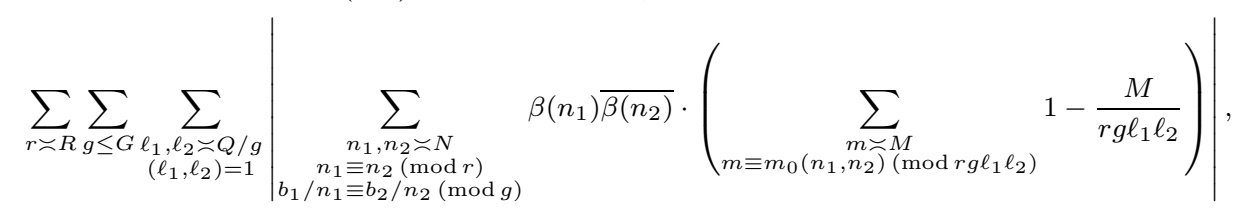

which, by (9.4), is

$$
\ll \sum_{r \asymp R} \sum_{g \leq G} \sum_{\substack{\ell_{1}, \ell_{2} \asymp Q / g \\\left(\ell_{1}, \ell_{2}\right)=1}} \sum_{\substack{0 \leq j \leq J \\ H_{j}:=2^{j} G / g}} \frac{1}{H_{j}} \sum_{1 \leq|h| \leq H_{j}}\left|\sum_{\substack{n_{1}, n_{2} \asymp N \\ n_{1} \equiv n_{2}(\bmod r) \\ n_{2} \equiv\left(b_{2} / b_{1}\right) n_{1}(\bmod g)}} \beta\left(n_{1}\right) \overline{\beta\left(n_{2}\right)} e_{r g \ell_{1} \ell_{2}}\left(m_{0}\left(n_{1}, n_{2}\right) h\right)\right| .
$$

We write $n_{1}=n, n_{2}=n+k r$, replace the $n_{2}$ variable with $k$, and define $m_{k}(n)=$ $m_{0}\left(n_{1}, n_{2}\right)$. To simplify matters, we shall proceed with $r, g, k$ and $j$ fixed, and then sum over these at the end, so we are reduced to studying

$$
\sum_{\substack{\ell_{1}, \ell_{2} \asymp L \\\left(\ell_{1}, \ell_{2}\right)=1}} \frac{1}{H} \sum_{1 \leq|h| \leq H} \sum_{\substack{n \asymp N \\=b_{1} k r(\bmod g)}} \beta(n) \overline{\beta(n+k r)} e_{r g \ell_{1} \ell_{2}}\left(m_{k}(n) h\right) \mid,
$$

where $L=Q / g$.

\section{LINNIK'S DISPERSION METHOD}

The proof of Zhang's theorem, and indeed of all the results in the literature of this type, use Linnik's dispersion method. The idea is to express the fact that $n$ belongs to an arithmetic progression using Fourier analysis; summing up over $n$ gives us a main term plus a sum of exponential sums, and then the challenge is to bound each of these exponential sums.

Often the sums come with weights, and judicious use of "Cauchying" allows one to work with an unweighted, but more complicated exponential sum. We will discuss bounds on exponential sums later in this section. These exponential sums are often Kloosterman sums, which one needs to bound. Individual Kloosterman sums can often be suitably bounded by Weil's or Deligne's theorem. However, sometimes one needs to get good bounds on averages of Kloosterman sums, a question that was brilliantly attacked by Deshouillers and Iwaniec in [11], using the (difficult) spectral theory of automorphic forms. Indeed all previous work breaking the $\sqrt{x}$ barrier (such as [17, [5]) uses these types of estimates. One of the remarkable aspects of Zhang's work is that he avoids these penible techniques and the restrictions that come with them.

Zhang was able to use only existing bounds on Kloosterman sums to prove his theorem, though he does use the sophisticated estimate of Birch and Bombieri 
from the appendix of 20]. Polymath8 indicates how even this deeper result can be avoided, so that the proof can be given using only "standard" estimates, which is what we do here. In order to get the strongest known version, one does need to involve these more difficult estimates, though they have been now reproved in an arguably more transparent way (see [42, [57]).

10.1. Removing the weights again. To remove the $\beta$ weights from (9.5), we begin by replacing the absolute value in (9.5) by the appropriate complex number $c_{h, \ell_{1}, \ell_{2}}$ of absolute value 1 and reorganize to obtain

$$
\sum_{\substack{n \asymp N \\\left(b_{2}-b_{1}\right)=\bar{\equiv}=\overline{\beta(n+k r)}}} \sum_{\substack{\ell_{1}, \ell_{2} \asymp L \\\left(\ell_{1}, \ell_{2}\right)=1}} \frac{1}{H} \sum_{1 \leq|h| \leq H} c_{h, \ell_{1}, \ell_{2}} e_{r g \ell_{1} \ell_{2}}\left(m_{k}(n) h\right) .
$$

We now "Cauchy" on the outer sum, which allows us to peel off the $\beta$ 's in the term

$$
\sum_{n}|\beta(n) \beta(n+k r)|^{2} \leq \sum_{n}|\beta(n)|^{4}=\|\beta\|_{4}^{4},
$$

times the more interesting term

$$
\sum_{n}\left|\sum_{\substack{\ell_{1}, \ell_{2} \asymp L \\\left(\ell_{1}, \ell_{2}\right)=1}} \frac{1}{H} \sum_{1 \leq|h| \leq H} c_{h, \ell_{1}, \ell_{2}} e_{r g \ell_{1} \ell_{2}}\left(m_{k}(n) h\right)\right|^{2} .
$$

We simply expand this sum and take absolute values for each fixed $h, j, \ell_{1}, \ell_{2}$, $m_{1}, m_{2}$, to obtain

$$
\leq \frac{1}{H^{2}} \sum_{1 \leq|h|,|j| \leq H_{i}} \sum_{\substack{\ell_{1}, \ell_{2}, m_{1}, m_{2} \asymp L \\\left(\ell_{1}, \ell_{2}\right)=\left(m_{1}, m_{2}\right)=1}}\left|\sum_{\substack{n \smile N \\\left(b_{2}-b_{1}\right)}} e_{r g \ell_{1} \ell_{2}}\left(m_{k}(n) h\right) e_{r g m_{1} m_{2}}\left(-m_{k}(n) j\right)\right| .
$$

Finally we have pure exponential sums, albeit horribly complicated, having bounded the contributions of the unspecified sequences $\alpha$ and $\beta$ to our original sums by surprisingly simple functions.

Our approach here works for a rather more general class of (pairs of) sequences $\alpha$ and $\beta$. However, in order to appropriately bound $\|\beta\|_{4}^{4}$ in terms of $\|\beta\|_{2}^{2}$ for the theorem formulated here, it is perhaps simplest to use the additional restriction on the size of each $\beta(n)$ that we gave here.

10.2. Exponential sums with complicated moduli. If $(r, s)=1$, then there are integers $a, b$ for which

$$
a r+b s=1 .
$$

Note that although there are infinitely many possibilities for the pair of integers $a, b$, the values of $a(\bmod s)$ and $b(\bmod r)$ are uniquely defined. If we divide the previous equation by $r s$, multiply by $m$, and then take $e($.$) of both sides, we obtain$

$$
e_{r s}(m)=e_{s}(a m) \cdot e_{r}(b m) .
$$

This allows us to write the exponential, in our last sum, explicitly. After some analysis, we find that the exponential sums at the end of the last subsection each 
take the form

$$
\sum_{\substack{n \asymp N \\ n \equiv a(\bmod q)}} e_{d_{1}}\left(\frac{C_{1}}{n}\right) e_{d_{2}}\left(\frac{C_{2}}{n+k r}\right)
$$

for some constants $C_{1}, C_{2}$ (where $d_{1}=r g\left[\ell_{1}, \ell_{2}\right], d_{2}=\left[m_{1}, m_{2}\right]$ and $q$ divides $g$ ). These constants depend on many variables but are independent of $n$. With a change of variable $n \mapsto q n+a$, we transform this to another sum of the same shape but now summing over all integers $n$ in a given interval.

10.3. Exponential sums: from the incomplete to the complete. We now have the sum of the exponential of a function of $n$, over the integers in an interval. There are typically many integers in this sum, so this is unlike what we encountered earlier (when we were summing 1). The terms of the sum are periodic of the period dividing $\left[d_{1}, d_{2}\right]$, and it is not difficult to sum the terms over a complete period. Hence we can restrict our attention to "incomplete sums" where the sum does not include a complete period.

We can now employ (9.3) once more. The coefficients $\hat{I}(h)$ are well understood, but the $\hat{f}(h)$ now take the form

$$
\sum_{n(\bmod q)} e_{d_{1}}\left(\frac{C_{1}}{n}+h n\right) e_{d_{2}}\left(\frac{C_{2}}{n+\Delta}+h n\right),
$$

a "complete" exponential sum.

The trick here is that we can factor the exponential into its prime factor exponentials, and then by the Chinese Remainder Theorem this sum equals the product over the primes $p$ dividing $q$ of the same sum but now over $n(\bmod p)$ with the appropriate $e_{p}(*)$. Hence we have reduced this question to asking for good bounds on exponential sums of the form

$$
\sum_{n(\bmod p)} e_{p}\left(\frac{a}{n}+\frac{b}{n+\Delta}+c n\right) .
$$

Here we omit values of $n$ for which a denominator is 0 . As long as this does not degenerate (for example, it would degenerate if $p \mid a, b, c$ ) then Weil's theorem implies that this is $\leq \kappa p^{1 / 2}$, for some constant $\kappa>0$. Therefore the complete sum over $n$ $(\bmod q)$ is $\leq \kappa^{\nu(q)} q^{1 / 2}$. This in turn allows us to bound our incomplete sum (10.2) and to bound the term at the end of the previous section.

The calculations to put this into practice are onerous, and we shall omit these details here. At the end one finds that the bounds deduced are acceptably small if

$$
x^{1 / 2} \geq N>x^{(2+\epsilon) / 5}
$$

where $\epsilon>12 \eta+7 \delta$. However this is not quite good enough, since we need to be able to take $N$ as small as $x^{1 / 3}$.

We can try a modification of this proof, the most successful being where, before we "Cauchy" equation (10.1), we also fix the $\ell_{1}$ variable. This variant allows us to extend our range to all

$$
N>x^{\frac{1}{3}+\epsilon},
$$

where $\epsilon>\frac{14}{3} \eta+\frac{7}{2} \delta$. We are very close to the exponent $\frac{1}{3}$, but it seems that we are destined to just fail. 


\section{Complete exponential sums: COMBINING INFORMATION THE GRAHAM-RINGROSE WAY}

The "square-root cancellation" for incomplete exponential sums of the form $\left|\sum_{n} e_{q}(f(n))\right|$ for various moduli $q$, with the sum over $n$ in an interval of length $N<q$, is not quite good enough to obtain our results.

Graham and Ringrose [26] proved that we can improve the (analogous) incomplete character sum bounds when $q$ is smooth. Here we follow Polymath8 [57, 21 who showed how to modify the Graham-Ringrose argument to incomplete exponential sums. This will allow us to reduce the size of $N$ in the above argument and prove our result.

11.1. Formulating the improved incomplete exponential sum result. For convenience we will write the entry of the exponential sum as $f(n)$, which should be thought of as taking the form $a / n+b /(n+\Delta)+c n$, though the argument is rather more general. We assume that $N<q$, so that the Weil bound gives

$$
\left|\sum_{n} e_{q}(f(n))\right| \ll \tau(q)^{A} q^{1 / 2}
$$

for some constant $A$ which depends only on the degree of $f$.

In what follows we will assume that $q$ is factored as $q=q_{1} q_{2}$, and we will deduce that

$$
\left|\sum_{n} e_{q}(f(n))\right| \ll\left(q_{1}^{1 / 2}+q_{2}^{1 / 4}\right) \tau(q)^{A}(\log q) N^{1 / 2} .
$$

If $q$ is $y$-smooth, then we let $q_{1}$ be the largest divisor of $q$ that is $\leq(q y)^{1 / 3}$ so that it must be $>\left(q / y^{2}\right)^{1 / 3}$, and so $q_{2} \leq(q y)^{2 / 3}$. Hence the last bound implies

$$
\left|\sum_{n} e_{q}(f(n))\right| \ll \tau(q)^{A}(q y)^{1 / 6}(\log q) N^{1 / 2} .
$$

It is this bound that we insert into the machinery of the previous section, and it allows us to extend our range to all

$$
N>x^{\frac{3}{10}+\epsilon},
$$

where $\epsilon$ is bounded below by a (positive) linear combination of $\eta$ and $\delta$. In order that we can stretch the range down to all $N>x^{\frac{1}{3}}$, this method requires that

$$
162 \eta+90 \delta<1 \text {. }
$$

11.2. Proof of (11.2). We may assume

$$
q_{1} \leq N \leq q_{2},
$$

else if $N<q_{1}$, we have the trivial bound $\leq N<\left(q_{1} N\right)^{1 / 2}$, and if $N>q_{2}$, then (11.1) implies the result since $q^{1 / 2}=\left(q_{1} q_{2}\right)^{1 / 2}<\left(q_{1} N\right)^{1 / 2}$.

The main idea will be to reduce our incomplete exponential sum $\bmod q$, to a sum of incomplete exponential sums $\bmod q_{2}$. Now

$$
e_{q}\left(f\left(n+k q_{1}\right)\right)=e_{q_{1}}\left(f(n) / q_{2}\right) e_{q_{2}}\left(f\left(n+k q_{1}\right) / q_{1}\right)
$$

\footnotetext{
$35]$.

${ }^{21}$ Who, in turn, essentially rediscovered an earlier argument of Heath-Brown (see section 9 of
} 
so that, by a simple change of variable, we have

$$
\sum_{n} e_{q}(f(n))=\sum_{n} e_{q}\left(f\left(n+k q_{1}\right)\right)=\sum_{n} e_{q_{1}}\left(f(n) / q_{2}\right) e_{q_{2}}\left(f\left(n+k q_{1}\right) / q_{1}\right) .
$$

Now, if we sum this over all $k, 1 \leq k \leq K:=\left\lfloor N / q_{1}\right\rfloor$, then we have

$$
K \sum_{n} e_{q}(f(n))=\sum_{n} e_{q_{1}}\left(f(n) / q_{2}\right) \sum_{k=1}^{K} e_{q_{2}}\left(f\left(n+k q_{1}\right) / q_{1}\right),
$$

and so

$$
\begin{aligned}
\left|K \sum_{n} e_{q}(f(n))\right|^{2} & \leq\left(\sum_{n}\left|\sum_{k=1}^{K} e_{q_{2}}\left(f\left(n+k q_{1}\right) / q_{1}\right)\right|\right)^{2} \\
& \ll N \sum_{n}\left|\sum_{k=1}^{K} e_{q_{2}}\left(f\left(n+k q_{1}\right) / q_{1}\right)\right|^{2} \\
& =N \sum_{1 \leq k, k^{\prime} \leq K} \sum_{n} e_{q_{2}}\left(g_{k, k^{\prime}}(n)\right),
\end{aligned}
$$

where $g_{k, k^{\prime}}(n):=\left(f\left(n+k q_{1}\right)-f\left(n+k^{\prime} q_{1}\right)\right) / q_{1}\left(\bmod q_{2}\right)$ if $n+k q_{1}, n+k^{\prime} q_{1} \in I$, and $g_{k, k^{\prime}}(n):=0$ otherwise. If $k=k^{\prime}$, then $g_{k, k}(n)=0$, and so these terms contribute $\leq K N^{2}$.

We now apply the bound of (11.1) taking $f=g_{k, k}$ for $k \neq k^{\prime}$. Calculating the sum yields (11.2).

11.3. Better results. In [57] the authors obtain better results using somewhat deeper techniques.

By replacing the set of $y$-smooth integers by the much larger class of integers with divisors in a prespecified interval (and such that those divisors have divisors in a different prespecified interval, etc., since one can iterate the proof in the previous section), they improve the restriction to

$$
84 \eta+48 \delta<1 .
$$

Following Zhang, they also gained bounds on certain higher-order convolutions (of the shape $\alpha * 1 * 1 * 1$ ), though here needing deeper exponential sum estimates, and they were then able to improve the restriction to (slightly better than)

$$
43 \eta+27 \delta<1
$$

11.4. Final remark. It is worth noting that one can obtain the same quality of results only assuming a bound $\ll p^{2 / 3-\epsilon}$ for the relevant exponential sums in finite fields.

\section{Further APplications}

Since Maynard's preprint appeared on arXiv, there has been a flowering of diverse applications of the techniques, to all sorts of questions from classical analytic number theory. My favorite (in that it is such a surprising application) was given by Pollack [55], who connected these ideas to another famous problem:

- In 1927 Artin conjectured that any integer $g$, which is not a perfect square and not -1 , is a primitive root for infinitely many distinct primes. Following beautiful work of Gupta and Murty [31, Heath-Brown 34] showed that this must be true 
for all but at most two primes $g$. Hooley [39] had shown how to prove Artin's conjecture assuming the Generalized Riemann Hypothesis. Pollack [55] has now shown, assuming the Generalized Riemann Hypothesis, that any such integer $g$ is a primitive root for each of infinitely $m$-tuples of primes which differ by no more than $B_{m}$.

Another beautiful result giving prime patterns:

- Combining ideas from this article with those from Green and Tao [29, Pintz showed in 51 that there exists an integer $B>0$ such that are infinitely many arithmetic progressions of primes $p_{n}, \ldots, p_{n+k}$ such that each of $p_{n}+B, \ldots, p_{n+k}+B$ is also prime.

12.1. Prime ideals. There are several analogous results in number fields.

- Thorner 64. showed that, for any given binary quadratic form $f$ of discriminant $D<0$, there are infinitely many pairs of distinct primes $p$ and $q=p+B(D)$ which are values of $f$.

Let $A$ be the ring of integers of a given number field $K$.

- Thorner 64] showed that if $K / \mathbb{Q}$ is Galois, then there are infinitely many pairs of prime ideals of $A$ whose norms are distinct primes that differ by $B(K)$.

- Castillo, Hall, Lemke Oliver, Pollack, and Thompson 8 proved that, for any admissible $k$-tuple $h_{1}, \ldots, h_{k}$ in $A$, there are infinitely many $\alpha \in A$ such that at least $m$ of the ideals $\left(\alpha+h_{1}\right), \ldots,\left(\alpha+h_{k}\right)$ are prime. Here we need $k$ to be sufficiently large as a function of $K$ and $m$.

12.2. Applications to (irreducible) polynomials. Castillo, Hall, Lemke Oliver, Pollack, and Thompson [8] also proved that, for any admissible $k$-tuple $h_{1}, \ldots, h_{k}$ of polynomials in $\mathbb{F}_{q}[t]$, there are infinitely many $f \in \mathbb{F}_{q}[t]$ such that at least $m$ of the polynomials $f+h_{1}, \ldots, f+h_{k}$ are irreducible in $\mathbb{F}_{q}[t]$. Here we need $k$ to be sufficiently large as a function of $m$ (but not $q$ ); in fact, it must be the same bound as was required in Maynard's result that was proved earlier.

12.3. Quadratic twists of elliptic curves and coefficients of modular forms. Thorner 64 gave several applications to elliptic curves and modular forms:

- For any given newform $f(z)=\sum_{n \geq 1} a_{f}(n) q^{n}$ for $\Gamma_{0}(N)$ of even weight $k \geq 2$ and any prime $\ell$, there exist infinitely many pairs of distinct primes $p$ and $q=$ $p+B(f, \ell)$ for which $a_{f}(p) \equiv a_{f}(q)(\bmod \ell)$.

- There exist infinitely many pairs of distinct primes $p$ and $q=p+B_{0}$ such that the elliptic curves $p y^{2}=x^{3}-x$ and $q y^{2}=x^{3}-x$ each have only finitely many rational points.

- There exist infinitely many pairs of distinct primes $p$ and $q=p+B_{1}$ such that the elliptic curves $p y^{2}=x^{3}-x$ and $q y^{2}=x^{3}-x$ each have infinitely many rational points.

These last two results can be generalized to other elliptic curves for which certain (technical) properties hold.

12.4. Quantitative forms of the Maynard-Tao theorem. These can give a lower bound for the number of $n \in[x, 2 x]$ for which at least $m$ of $n+a_{1}, \ldots, n+a_{k}$ are prime, and also they can allow the $a_{j}$ to vary with $x$, getting as large as a multiple of $\log x$. Maynard [47] generalized his result and proof, which were discussed earlier in this article, as follows: 
- Suppose that we are given a finite admissible set of linear forms $\left\{b_{j} x+a_{j}\right\}$, a sequence of integers $\mathcal{N}$ which is "well distributed" in arithmetic progressions on average, and a set of primes $\mathcal{P}$ such that each $\left\{b_{j} n+a_{j}: n \in \mathcal{N}\right\} \cap \mathcal{P}$ is also "well distributed" in arithmetic progressions on average. Then there are infinitely many integers $n \in \mathcal{N}$ such that at least $\ell$ of the $\left\{b_{j} n+a_{j}: 1 \leq j \leq k\right\}$ are primes in $\mathcal{P}$, where $\ell \gg \log k$. (This result can be used to deduce several of the others listed here.)

- One amazing consequence of this result is that, for any $x, y \geq 1$, there are $\gg x \exp (-\sqrt{\log x})$ integers $n \in(x, 2 x]$ for which:

There are $\gg \log y$ primes in the interval $(n, n+y]$.

- Let $d_{n}=p_{n+1}-p_{n}$ where $p_{n}$ is the $n$th smallest prime. Pintz [52] showed that there are infinitely many $n$ for which $d_{n}, d_{n+k}$ are significantly larger than $\log n$, whereas each of $d_{n+1}, \ldots, d_{n+k-1}$ are bounded.

12.5. The set of limit points $\mathcal{L}$ of the $\frac{p_{n+1}-p_{n}}{\log p_{n}}$. It is conjectured that $\mathcal{L}=[0, \infty]$. The result of GPY gave that $0 \in L$, and it has long been known that $\infty \in L$ (see Appendix B).

- The quantitative form of the Maynard-Tao theorem immediately gives that if $B$ is a set of any 50 positive real numbers, then $(B-B)^{+}$contains an element of $L$. From this one can deduce [1] that the measure of $L \cap[0, x]$ is at least $x / 49$.

- Pintz [51] showed that $[0, c] \subset L$ for some $c>0$ (though the proof does not yield a value for $c$ ).

\section{ApPEndix A.}

\section{HARDY AND LiTTLEWOOD'S HEURISTIC FOR THE TWIN PRIME CONJECTURE}

The rather elegant and natural heuristic for the quantitative twin prime conjecture, which we described in Section 2.5 was not the original way in which Hardy and Littlewood made this extraordinary prediction. The genesis of their technique lies in the circle method, which they developed together with Ramanujan. The idea is that one can distinguish the integer 0 from all other integers, since

$$
\int_{0}^{1} e(n t) d t= \begin{cases}1 & \text { if } n=0 \\ 0 & \text { otherwise }\end{cases}
$$

where, for any real number $t$, we write $e(t):=e^{2 \pi i t}$. Notice that this is literally an integral around the unit circle. Therefore, to determine whether the two given primes $p$ and $q$ differ by 2 , we simply determine

$$
\int_{0}^{1} e((p-q-2) t) d t
$$

If we sum this up over all $p, q \leq x$, we find that the number of twin primes $p, p+2 \leq x$ equals, exactly,

$$
\sum_{\substack{p, q \leq x \\ p, q \text { primes }}} \int_{0}^{1} e((p-q-2) t) d t=\int_{0}^{1}|P(t)|^{2} e(-2 t) d t, \text { where } P(t):=\sum_{\substack{p \leq x \\ p \text { prime }}} e(p t) .
$$

In the circle method one next distinguishes between those parts of the integral which are large (the major arcs), and those that are small (the minor arcs). Typically, the major arcs are small arcs around those $t$ that are rationals with small denominators. 
Here the width of the arc is about $1 / x$, and we wish to understand the contribution at $t=a / m$, where $(a, m)=1$. We then have

$$
P(a / m)=\sum_{\substack{b(\bmod m) \\(b, m)=1}} e_{m}(a b) \pi(x ; m, b),
$$

where $e_{m}(b)=e\left(\frac{b}{m}\right)=e^{2 \pi i b / m}$. We note the easily proved identity

$$
\sum_{r(\bmod m),(r, m)=1} e_{m}(r k)=\phi((k, m)) \mu(m /(m, k)) .
$$

Assuming the prime number theorem for arithmetic progressions with a good error term, we therefore see that

$$
P(a / m) \approx \frac{x}{\phi(m) \log x} \sum_{\substack{(\bmod m) \\(b, m)=1}} e_{m}(a b)=\frac{\mu(m)}{\phi(m)} \frac{x}{\log x} .
$$

Hence, in total we predict that the number of prime pairs $p, p+2 \leq x$ is roughly

$$
\begin{aligned}
& \approx \frac{1}{x} \sum_{m \leq M} \sum_{a:(a, m)=1} e_{m}(-2 a)\left|\frac{\mu(m)}{\phi(m)} \frac{x}{\log x}\right|^{2} \\
& \approx \frac{x}{(\log x)^{2}} \sum_{m \geq 1} \frac{\mu(m)^{2}}{\phi(m)^{2}} \cdot \phi((2, m)) \mu(m /(2, m)) \\
& =\frac{x}{(\log x)^{2}}\left(1+\frac{1}{\phi(2)}\right) \prod_{p>2}\left(1-\frac{1}{\phi(p)^{2}}\right)=C \frac{x}{(\log x)^{2}} ;
\end{aligned}
$$

the same prediction as we obtained in Section 2.5 by a very different heuristic. Moreover, an analogous argument yields the more general conjecture for prime pairs $p, p+h$.

Why doesn't this argument lead to a proof of the twin prime conjecture? For the moment we have little idea how to show that the minor arcs contribute very little. We know that the minor arcs can be quite large in absolute value, so to prove the twin prime conjecture, we would have to find cancelation in the arguments of the integrand on the minor arcs. Indeed it is an important open problem to find cancelation in the minor arcs in any problem.

However, if we add more variables, then appropriate modifications of this argument can be made to work. Indeed it is this kind of circle method argument that led to Helfgott's recent proof [36] that every odd integer $\geq 3$ is the sum of no more than three primes.

ApPEndix B.

\section{Stop The PRESS! LARGE GAPS BETWEen PRIMES}

The average gap between primes $\leq x$ is about $\log x$. This article has focused on recent work to prove that there are many much smaller gaps. How about larger gaps? Can one prove that there are infinitely many gaps between consecutive primes that are much larger than $\log x$ ? In 1931, Westzynthius showed that for any constant $C>0$ there exist infinitely $n$ for which $p_{n+1}-p_{n}>C \log p_{n}$. His idea is simply to find many consecutive integers, each of which has a very small prime factor (so none of these integers can be a prime). Erdős and Rankin developed this 
method improving the result to the following. There exists a constant $C>0 \mathrm{such}$ that there are infinitely $n$ for which

$$
p_{n+1}-p_{n}>C \log p_{n} \frac{\log \log p_{n}}{\left(\log \log \log p_{n}\right)^{2}} \log \log \log \log p_{n} .
$$

Subsequent papers increased the constant $C$, though they were unable to show that one could take arbitrarily large $C$ (and Cramer conjectured that gaps can be much larger, even as large as $\left.\left(\log p_{n}\right)^{2}\right)$. Erdös liked to stimulate research on his favorite questions by offering cash prizes. The largest prize he ever offered was $\$ 10,000$ to whoever could show that one can take $C$, here, to be arbitrarily large.

The GPY method was developed to prove that there are (far) smaller gaps between primes than the average. It came as quite a surprise when, in August 2014, James Maynard [4] showed that one could ingeniously modify the argument for small prime gaps, to improve the Erdös-Rankin theorem for large prime gaps, not only getting arbitrarily large $C$ but replacing $C$ by something like $\log \log \log p_{n}$. The same week, Ford, Green, Konyagin, and Tao [13] modified the Erdös-Rankin argument very differently, the main ingredient being a version of Green and Tao's [29] famous theorem on $k$-term arithmetic progressions of primes, to also show that one could take $C$ to be arbitrarily large.

It is an exciting time for gaps between primes.

\section{ACKNOWLEDGMENTS}

The author thanks Sary Drappeau, Tristan Freiberg, John Friedlander, Adam Harper, Dimitris Koukoulopoulos, Emmanuel Kowalski, James Maynard, Pace Nielsen, Robert Lemke Oliver, Terry Tao, and the anonymous referee for their comments on earlier versions of this article.

\section{ABOUt THE AUTHOR}

Andrew Granville is the Canada Research Chair in number theory at the University of Montreal. He works mainly in analytic number theory, in recent years developing the "pretentious approach" with K. Soundararajan, which differs from Riemann's approach by not considering the zeros of zeta-functions. He has helped quite a few students and post-docs, including James Maynard in 2013-14, during the time in which he made his extraordinary contribution detailed in this article.

\section{REFERENCES}

[1] W. D. Banks, T. Freiberg, J. Maynard, On limit points of the sequence of normalized prime gaps, preprint.

[2] W. D. Banks, T. Freiberg, C. L. Turnage-Butterbaugh, Consecutive primes in tuples, preprint.

[3] E. Bombieri, On the large sieve, Mathematika 12 (1965), 201-225. MR0197425 (33 \#5590)

[4] E. Bombieri and H. Davenport, Small differences between prime numbers, Proc. Roy. Soc. Ser. A 293 (1966), 1-18. MR0199165 (33 \#7314)

[5] E. Bombieri, J. B. Friedlander, and H. Iwaniec, Primes in arithmetic progressions to large moduli, Acta Math. 156 (1986), no. 3-4, 203-251, DOI 10.1007/BF02399204. MR834613 (88b:11058)

[6] E. Bombieri, J. B. Friedlander, and H. Iwaniec, Primes in arithmetic progressions to large moduli. II, Math. Ann. 277 (1987), no. 3, 361-393, DOI 10.1007/BF01458321. MR891581 (88f:11085)

[7] E. Bombieri, J. B. Friedlander, and H. Iwaniec, Primes in arithmetic progressions to large moduli. III, J. Amer. Math. Soc. 2 (1989), no. 2, 215-224, DOI 10.2307/1990976. MR976723 (89m:11087) 
[8] A. Castillo, C. Hall, R. J. Lemke Oliver, P. Pollack, L. Thompson, Bounded gaps between primes in number fields and function fields, preprint.

[9] L. Chua, S. Park, G. D. Smith, Bounded gaps between primes in special sequences, preprint.

[10] Pierre Deligne, La conjecture de Weil. II (French), Inst. Hautes Études Sci. Publ. Math. 52 (1980), 137-252. MR601520(83c:14017)

[11] J.-M. Deshouillers and H. Iwaniec, Kloosterman sums and Fourier coefficients of cusp forms, Invent. Math. 70 (1982/83), no. 2, 219-288, DOI 10.1007/BF01390728. MR684172 $(84 \mathrm{~m}: 10015)$

[12] P. D. T. A. Elliott and H. Halberstam, A conjecture in prime number theory, Symposia Mathematica, Vol. IV (INDAM, Rome, 1968/69), Academic Press, London, 1970, pp. 59-72. MR.0276195 (43 \#1943)

[13] K. Ford, B. Green, S. Konyagin and T. Tao, Large gaps between consecutive prime numbers, preprint.

[14] E. Fouvry, A new form of the error term in the linear sieve, Acta Arith., 37 (1980), 307-320.

[15] Étienne Fouvry, Autour du théorème de Bombieri-Vinogradov (French), Acta Math. 152 (1984), no. 3-4, 219-244, DOI 10.1007/BF02392198. MR741055 (85m:11052)

[16] E. Fouvry and H. Iwaniec, On a theorem of Bombieri-Vinogradov type, Mathematika 27 (1980), no. 2, 135-152 (1981), DOI 10.1112/S0025579300010032. MR610700 (82h:10057)

[17] E. Fouvry and H. Iwaniec, Primes in arithmetic progressions, Acta Arith. 42 (1983), no. 2, 197-218. MR719249 (84k:10035)

[18] T. Freiberg, A note on the theorem of Maynard and Tao, preprint.

[19] John Friedlander and Andrew Granville, Limitations to the equi-distribution of primes. I, Ann. of Math. (2) 129 (1989), no. 2, 363-382, DOI 10.2307/1971450. MR986796 (90e:11125)

[20] John B. Friedlander and Henryk Iwaniec, Incomplete Kloosterman sums and a divisor problem, Ann. of Math. (2) 121 (1985), no. 2, 319-350, DOI 10.2307/1971175. With an appendix by Bryan J. Birch and Enrico Bombieri. MR786351 (86i:11050)

[21] J. Friedlander and H. Iwaniec, Close encounters among the primes, preprint.

[22] P. X. Gallagher, Bombieri's mean value theorem, Mathematika 15 (1968), 1-6. MR.0237442 (38 \#5724)

[23] Daniel A. Goldston, János Pintz, and Cem Y. Yıldırım, Primes in tuples. I, Ann. of Math. (2) 170 (2009), no. 2, 819-862, DOI 10.4007/annals.2009.170.819. MR2552109 (2011c:11146)

[24] D. A. Goldston, S. W. Graham, J. Pintz, and C. Y. Yıldırım, Small gaps between primes or almost primes, Trans. Amer. Math. Soc. 361 (2009), no. 10, 5285-5330, DOI 10.1090/S00029947-09-04788-6. MR2515812(2010d:11108)

[25] Solomon Wolf Golomb, Problems in the distribution of the prime numbers, ProQuest LLC, Ann Arbor, MI, 1957. Thesis (Ph.D.)-Harvard University. MR2938872

[26] S. W. Graham and C. J. Ringrose, Lower bounds for least quadratic nonresidues, Analytic number theory (Allerton Park, IL, 1989), Progr. Math., vol. 85, Birkhäuser Boston, Boston, MA, 1990, pp. 269-309. MR1084186 (92d:11108)

[27] A. Granville, D.M. Kane, D. Koukoulopoulos, and R. Lemke Oliver, Best possible densities, as a consequence of Zhang-Maynard-Tao, to appear.

[28] A. Granville and K. Soundararajan, Multiplicative number theory; the alternative approach, to appear.

[29] Ben Green and Terence Tao, The primes contain arbitrarily long arithmetic progressions, Ann. of Math. (2) 167 (2008), no. 2, 481-547, DOI 10.4007/annals.2008.167.481. MR2415379 (2009e:11181)

[30] Ben Green, Terence Tao, and Tamar Ziegler, An inverse theorem for the Gowers $U^{s+1}[N]$ norm, Ann. of Math. (2) 176 (2012), no. 2, 1231-1372, DOI 10.4007/annals.2012.176.2.11. MR2950773

[31] Rajiv Gupta and M. Ram Murty, A remark on Artin's conjecture, Invent. Math. 78 (1984), no. 1, 127-130, DOI 10.1007/BF01388719. MR762358 (86d:11003)

[32] G. H. Hardy and J. E. Littlewood, Some problems of 'Partitio numerorum'; III: On the expression of a number as a sum of primes, Acta Math. 44 (1923), no. 1, 1-70, DOI 10.1007/BF02403921. MR1555183

[33] D. R. Heath-Brown, Prime numbers in short intervals and a generalized Vaughan identity, Canad. J. Math. 34 (1982), no. 6, 1365-1377, DOI 10.4153/CJM-1982-095-9. MR678676 (84g:10075) 
[34] D. R. Heath-Brown, Artin's conjecture for primitive roots, Quart. J. Math. Oxford Ser. (2) 37 (1986), no. 145, 27-38, DOI 10.1093/qmath/37.1.27. MR830627(88a:11004)

[35] D. R. Heath-Brown, The largest prime factor of $X^{3}+2$, Proc. London Math. Soc. (3) 82 (2001), no. 3, 554-596, DOI 10.1112/plms/82.3.554. MR1816689 (2001m:11158)

[36] H. A. Helfgott, Major arcs for Goldbach's theorem, to appear.

[37] Douglas Hensley and Ian Richards, On the incompatibility of two conjectures concerning primes, Analytic number theory (Proc. Sympos. Pure Math., Vol. XXIV, St. Louis Univ., St. Louis, Mo., 1972), Amer. Math. Soc., Providence, R.I., 1973, pp. 123-127. MR0340194 (49 \#4950)

[38] Douglas Hensley and Ian Richards, Primes in intervals, Acta Arith. 25 (1973/74), 375-391. MR0396440 (53 \#305)

[39] Christopher Hooley, On Artin's conjecture, J. Reine Angew. Math. 225 (1967), 209-220. MR0207630(34 \#7445)

[40] Henryk Iwaniec and Emmanuel Kowalski, Analytic number theory, American Mathematical Society Colloquium Publications, vol. 53, American Mathematical Society, Providence, RI, 2004. MR2061214 (2005h:11005)

[41] H. D. Kloosterman, On the representation of numbers in the form $a x^{2}+b y^{2}+c z^{2}+d t^{2}$, Acta Mathematica 49 (1926), pp. 407-464.

[42] E. Kowalski, Gaps between prime numbers and primes in arithmetic progressions (after $Y$. Zhang and J. Maynard), Séminaire Bourbaki 66 (2013-2014), no. 1084.

[43] H. Li, H. Pan, Bounded gaps between primes of the special form, preprint.

[44] Helmut Maier, Small differences between prime numbers, Michigan Math. J. 35 (1988), no. 3, 323-344, DOI 10.1307/mmj/1029003814. MR.978303 (90e:11126)

[45] J. Maynard, Small gaps between primes, to appear, Annals Math.

[46] J. Maynard, Large gaps between primes, preprint.

[47] J. Maynard, Dense clusters of primes in subsets, preprint.

[48] L. J. Mordell, On a sum analogous to a Gauss's sum, Quart. J. Math. Oxford Ser. 3 (1932), 161-167.

[49] Yoichi Motohashi, An induction principle for the generalization of Bombieri's prime number theorem, Proc. Japan Acad. 52 (1976), no. 6, 273-275. MR0422179 (54 \#10171)

[50] Yoichi Motohashi and János Pintz, A smoothed GPY sieve, Bull. Lond. Math. Soc. 40 (2008), no. 2, 298-310, DOI 10.1112/blms/bdn023. MR2414788(2009d:11132)

[51] J. Pintz, Polignac Numbers, Conjectures of Erdős on Gaps between Primes, Arithmetic Progressions in Primes, and the Bounded Gap Conjecture, preprint.

[52] J. Pintz, On the ratio of consecutive gaps between primes, preprint.

[53] J. Pintz, On the distribution of gaps between consecutive primes, preprint.

[54] A. De Polignac, Six propositions arithmologiques déduites du crible d'Ératosothène, Nouvelles annales de mathématiques 8 (1849), 423-429.

[55] P. Pollack, Bounded gaps between primes with a given primitive root, preprint.

[56] P. Pollack and L. Thompson, Arithmetic functions at consecutive shifted primes, preprint.

[57] D. H. J. Polymath, New equidistribution estimates of Zhang type, preprint.

[58] D. H. J. Polymath, Variants of the Selberg sieve, and bounded intervals containing many primes, preprint.

[59] A. Schinzel, Remarks on the paper "Sur certaines hypothèses concernant les nombres premiers", Acta Arith. 7 (1961/1962), 1-8. MR0130203(24 \#A70)

[60] A. Selberg, On elementary methods in prime number theory and their limitations, in Proc. 11th Scand. Math. Cong. Trondheim (1949), Collected Works, Vol. I, 388-477, SpringerVerlag, Berlin-Göttingen-Heidelberg, 1989.

[61] P. Shiu, A Brun-Titchmarsh theorem for multiplicative functions, J. Reine Angew. Math. 313 (1980), 161-170, DOI 10.1515/crll.1980.313.161. MR552470(81h:10065)

[62] K. Soundararajan, Small gaps between prime numbers: the work of Goldston-Pintz-Yıldırım, Bull. Amer. Math. Soc. (N.S.) 44 (2007), no. 1, 1-18, DOI 10.1090/S0273-0979-06-01142-6. MR2265008(2007k:11150)

[63] T. Tao, private communication.

[64] J. Thorner, Bounded gaps between primes in Chebotarev sets, preprint.

[65] Robert C. Vaughan, Sommes trigonométriques sur les nombres premiers (French, with English summary), C. R. Acad. Sci. Paris Sér. A-B 285 (1977), no. 16, A981-A983. MR0498434 $(58 \# 16555)$ 
[66] A. I. Vinogradov, The density hypothesis for Dirichet L-series (Russian), Izv. Akad. Nauk SSSR Ser. Mat. 29 (1965), 903-934. MR0197414 (33 \#5579)

[67] André Weil, Numbers of solutions of equations in finite fields, Bull. Amer. Math. Soc. 55 (1949), 497-508. MR0029393 (10,592e)

[68] Yitang Zhang, Bounded gaps between primes, Ann. of Math. (2) 179 (2014), no. 3, 1121-1174, DOI 10.4007/annals.2014.179.3.7. MR3171761

DÉPartement de mathématiques et de statistiques, Université de Montréal, Montréal QC H3C 3J7, CANADA

E-mail address: andrew@dms.umontreal.ca 